\title{
Convergence of Non-autonomous Attractors for Subquintic Weakly Damped Wave Equation
}

\author{
Jakub Banaśkiewicz ${ }^{1}$ (D) Piotr Kalita ${ }^{1}$
}

Accepted: 25 May 2021 / Published online: 8 June 2021

(C) The Author(s) 2021

\begin{abstract}
We study the non-autonomous weakly damped wave equation with subquintic growth condition on the nonlinearity. Our main focus is the class of Shatah-Struwe solutions, which satisfy the Strichartz estimates and coincide with the class of solutions obtained by the Galerkin method. For this class we show the existence and smoothness of pullback, uniform, and cocycle attractors and the relations between them. We also prove that these non-autonomous attractors converge upper-semicontinuously to the global attractor for the limit autonomous problem if the time-dependent nonlinearity tends to a time independent function in an appropriate way.
\end{abstract}

\section{Introduction}

In this paper we are interested in the existence, regularity and upper-semicontinuous convergence of pullback, uniform and cocycle attractors of the problems governed by the following family of weakly damped wave equations

$$
u_{t t}+u_{t}-\Delta u=f_{\varepsilon}(t, u) .
$$

We prove that these attractors converge as $\varepsilon \rightarrow 0$ to the global attractor of the problem governed by the limit autonomous equation

This work was supported by National Science Center (NCN) of Poland under Projects No. DEC-2017/25/B/ST1/00302 and UMO-2016/22/A/ST1/00077. We thank the reviewers for their comments which significantly improved the manuscript.

$\bowtie \quad$ Jakub Banaśkiewicz

Jakub.Banaskiewicz@im.uj.edu.pl

Piotr Kalita

piotr.kalita@ii.uj.edu.pl

1 Faculty of Mathematics and Computer Science, Jagiellonian University, ul. Łojasiewicza 6, 30-348 Kraków, Poland 


$$
u_{t t}+u_{t}-\Delta u=f_{0}(u)
$$

where $f_{\varepsilon} \rightarrow f_{0}$ in an appropriate sense. The unknowns are the functions $u:\left[t_{0}, \infty\right) \times$ $\Omega \rightarrow \mathbb{R}$, where $\Omega$ is an open and bounded domain in $\mathbb{R}^{3}$ with smooth boundary.

The theory of global attractors for the wave equation with damping term $u_{t}$ has been developed by Babin and Vishik [3], Ghidaglia and Temam [20], Hale [21], Haraux [23,24], Pata and Zelik [33]. Overview of the theory can be found, among others, in the monographs of Babin and Vishik [4], Haraux [25], Chueshov and Lasiecka [16]. We also mention the classical monographs of Henry [26], Hale [22], Robinson [34], Temam [41], and Dłotko and Cholewa [18] on infinite dimensional autonomous dynamical systems. Various types of non-autonomous attractors and their properties have been studied, among others, by Chepyzhov and Vishik [15], Cheban [13], Kloeden and Rasmussen [28], Carvalho et al. [11], Chepyzhov [14], and Bortolan et al. [8].

The existence of the global attractor for (1.2) with the cubic growth condition

$$
\left|f_{0}(s)\right| \leq C\left(1+|s|^{3}\right)
$$

has been obtained by Arrieta et al. [2]. This growth exponent had long been considered as critical. In 2016 Kalantarov et al. [27] used the findings on the Strichartz estimates for the wave equation on bounded domains $[6,9]$ to obtain the global attractor existence for the so called Shatah-Struwe solutions of quintic weakly damped wave equation, i.e. where the exponent 3 in (1.3) is replaced by 5 . These findings led to the rapid development of the theory for weakly damped wave equation with supercubic growth. In particular, global attractors for Shatah-Struwe solutions for supercubic case with forcing in $H^{-1}$ have been studied by Liu et al. [29], and the exponential attractors were investigated by Meng and Liu in [32]. We also mention the work [10] of Carvalho, Cholewa, and Dłotko who proved an existence of the weak global attractor for a concept of solutions for supercubic but subquintic case. Finally, the results on attractors for autonomous problems with supercubic nonlinearities have been generalized to the case of damping given by the fractional Laplacian in the subquintic case in [35] and in the quintic case in [36].

For a non-autonomous dynamical system there exist several important concepts of attractors: the pullback attractor, a time-dependent family of compact sets attracting "from the past" $[11,28]$, the uniform attractor, the minimal compact set attracting forwards in time uniformly with respect to the driven system of non-autonomous terms [15], and the cocycle attractor which, in a sense unifies and extends the last two concepts [7,28]. An overview of these notions can be found in the review article [5]. Recent intensive research on the characterization of pullback attractors and continuity properties for PDEs [7,11,28] has led to the results on the link between the notions of uniform, pullback, and cocycle attractors, namely an internal characterization of the uniform attractor as the union of the pullback attractors related to all their associated symbols (see [7], and Theorem 6.5 below), and thus allowing to define the notion of lifted invariance (see [7], and Definition 6.6 and Theorem 6.7 below) for uniform attractors.

There are several recent results on the non-autonomous version of the weakly damped wave equation with quintic, or at least supercubic, growth condition which 
use the concept of Shatah-Struwe solutions. Savostianov and Zelik in the article [37] obtained the existence of the uniform attractor for the problem governed by

$$
u_{t t}+u_{t}+(1-\Delta) u+f(u)=\mu(t),
$$

on the three dimensional torus, where $\mu(t)$ can be a measure. Mei, Xiong, and Sun [31] obtained the existence of the pullback attractor for the problem governed by the equation

$$
u_{t t}+u_{t}-\Delta u+f(u)=g(t),
$$

for the subquintic case on the space domain given by whole $\mathbb{R}^{3}$ in the so called locally uniform spaces. Mei and Sun [30] obtained the existence of the uniform attractor for non a translation compact forcing term for the problem governed by (1.4) with subquintic $f$. Finally, Chang, Li, Sun, and Zelik [12] considered the problem of the form

$$
u_{t t}+\gamma(t) u_{t}-\Delta u+f(u)=g
$$

and showed the existence of several types of non-autonomous attractors with quintic nonlinearity for the case where the damping may change sign. None of these results considered the nonlinearity of the form $f(t, u)$ and none of these results fully explored the structure of non-autonomous attractors and relation between pullback, uniform, and cocycle attractors characterized in [7]. The present paper aims to fill this gap.

In this article we generalize the results of [27] to the problem governed by the weakly damped non-autonomous wave equation (1.1) with the semilinear term $f_{\varepsilon}(t, u)$ which is a perturbation of the autonomous nonlinearity $f_{0}(u)$, cf. assumptions $(\mathrm{H} 2)$ and $(\mathrm{H} 3)$ in Sect. 3. We stress that we deal only with the case of the subquintic growth

$$
\left|\frac{\partial f_{\varepsilon}}{\partial u}(t, u)\right| \leq C\left(1+|u|^{4-\kappa}\right),
$$

for which we prove the results on the existence and asymptotic smoothness of ShatahStruwe solutions, derive the asymptotic smoothing estimates and obtain the result on the upper-semicontinuous convergence of attractors. Thus we extend and complete the previous results in [27] where only the autonomous case was considered, and in [30,31] where the nonlinearity was only in the autonomous term. We stress some key difficulties and achievements of our work. We follow the methodology of [27, Proposition 3.1 and Proposition 4.1] to derive the Strichartz estimate for the nonlinear problem from the one for the linear problem (where we use the continuation principle that can be found for example in [40, Proposition 1.21]) but in the proof we need the extra property that the constant $C_{h}$ in the linear Strichartz estimate

$$
\|u\|_{L^{4}\left(0, h ; L^{12}\right)} \leq C_{h}\left(\left\|\left(u_{0}, u_{1}\right)\right\|_{\mathcal{E}_{0}}+\|G\|_{L^{1}\left(0, T ; L^{2}\right)}\right)
$$

is a nondecreasing function of $h$. We establish this fact with the use of the ChristKiselev Lemma [38, Lemma 3.1]. Moreover, we define the weak solutions as the 
limits of the Galerkin approximations. In [27, Sect. 3] the authors work with the Shatah-Struwe solutions (i.e. the weak solutions posessing the extra $L^{4}\left(0, T ; L^{12}(\Omega)\right)$ regularity), and they prove that such solutions are indeed the limits of the Galerkin approximations, cf. [27, Corollary 3.6]. We establish that in the subcritical case the two notions are in fact equivalent, cf. our Lemma 5.9.

Main results of our paper are contained in Sects. 6 and 7. The main result of Sect. 6 is Theorem 6.10 on the existence and smoothness of uniform and cocycle attractors for the considered problem and the relation between the two notions. The key property needed for the existence of these objects is the uniform asymptotic smoothness obtained in Lemma 6.8. In [27, Corollary 4.3] the authors derive only $\mathcal{E}_{\delta}=$ $\left(H^{1+\delta} \cap H_{0}^{1}\right) \times H^{\delta}$ estimates for the autonomous case, with small $\delta>0$, mentioning in Remark 4.6 the possibility of using further bootstrapping arguments. We derive in Lemma 6.8 the relevant asymptotic smoothing estimates in $\mathcal{E}_{1}=\left(H^{2} \cap H_{0}^{1}\right) \times H_{0}^{1}$. While $\mathcal{E}_{\delta}$ estimates are sufficient for the attractor existence, our estimates allow us to deduce its regularity, namely the fact that it belongs to $\mathcal{E}_{1}$. Once we have the uniform asymptotic smoothing estimates we can use the recent findings of of [7,28], cf. [7, Theorem 3.12.], reminded here as Theorem 6.5 below. This abstract result is applied to get our Theorem 6.10 where we establish the existence of the uniform attractor $\mathcal{A}_{\varepsilon}$, and the cocycle attractor $\left\{\mathcal{A}_{\varepsilon}\left(p_{\varepsilon}\right)\right\}_{p_{\varepsilon} \in \mathcal{H}\left(f_{\varepsilon}\right)}$, an object parameterised by elements $p_{\varepsilon} \in \mathcal{H}\left(f_{\varepsilon}\right)$ of the hull of the time shifts of the translation compact non-autonomous term $f_{\varepsilon}$. Apart from the existence, application of [7, Theorem 3.12.] allows us to get the relation between the two objects, namely that

$$
\mathcal{A}_{\varepsilon}=\bigcup_{p_{\varepsilon} \in \mathcal{H}\left(f_{\varepsilon}\right)} \mathcal{A}\left(p_{\varepsilon}\right) .
$$

Finally, another novelty of the present paper is the upper-semicontinuity result of Sect. 7. In Theorem 7.4 we obtain that uniform attractors $\mathcal{A}_{\varepsilon}$ converge uppersemicontinuously to the limit attractor of the autonomous problem, i.e., that

$$
\lim _{\varepsilon \rightarrow 0^{+}} \operatorname{dist}_{\mathcal{E}_{0}}\left(\mathcal{A}_{\varepsilon}, \mathcal{A}_{0}\right)=0
$$

where dist $\mathcal{E}_{0}$ is the Hausdorff semidistance in the space $\mathcal{E}_{0}=H_{0}^{1} \times L^{2}$. The key role in the proof is played by the lifted-invariance property of the uniform attractor, cf. Definition 6.6 and Theorem 6.7, and uniform (with respect to $\varepsilon$ ) $\mathcal{E}_{1}$ boundedness of the uniform attractors $\mathcal{A}_{\varepsilon}$ obtained in Sect. 6. Note that due to (1.5) the obtained upper-semicontinuity result automatically allows us to deduce that

$$
\lim _{\varepsilon \rightarrow 0^{+}} \operatorname{dist}_{\mathcal{E}_{0}}\left(\mathcal{A}_{\varepsilon}\left(p_{\varepsilon}\right), \mathcal{A}_{0}\right)=0
$$

for every $\left\{p_{\varepsilon} \in \mathcal{H}\left(f_{\varepsilon}\right)\right\}_{\varepsilon \in[0,1]}$, i.e. that the cocycle (and hence also the pullback) attractors converge to the limit global attractor in the upper-semicontinuous sense.

The possible extension of our results involves dealing with a non-autonomous nonlinearity with critical quintic growth condition. This case is more delicate because the control of the energy norm of the initial data does not give the control over 
norm $L^{4}\left(0, T ; L^{12}(\Omega)\right)$ of the solution. To overcome this problem Kalantarov, Savostianov, and Zelik in [27] used the technique of trajectory attractors. Another interesting question is the possibility of extending the results of [19] about the convergence of non-autonomous attractors for equations

$$
\varepsilon u_{t t}+u_{t}-\Delta u=f_{\varepsilon}(t, u)
$$

to the attractor for the semilinear heat equation as $\varepsilon \rightarrow 0$, in the case of subquintic or quintic growth condition on $f$. The main difficulty is to obtain uniform Strichartz estimates with respect to $\varepsilon$. Finally we mention the possible further line of research involving the lower semicontinuous convergence of attractor and the stability of the attractor structure under perturbation.

The structure of the article is as follows. After some preliminary facts are reminded in Sect. 2, the formulation of the problem, assumptions of its data, and some auxiliary results regarding the translation compactness of the non-autonomous term are presented in Sect. 3. Next, Sect. 4 is devoted to the Galerkin solutions and their dissipativity. The following Sect. 5 contains the results on the Strichartz estimates, Shatah-Struwe solutions, and their equivalence with the Galerkin solutions. The result on the existence and asymptotic smoothness of non-autonomous attractors, Theorem 6.10, is contained in Sect. 6, while in Sect. 7 we prove their upper-semicontinuous convergence to the global attractor of the limit autonomous problem.

\section{Preliminaries}

Let $\Omega \subset \mathbb{R}^{3}$ be a bounded and open set with sufficiently smooth boundary. We will use the notation $L^{2}$ for $L^{2}(\Omega)$ and, in general, for notation brevity, we will skip writing dependence on $\Omega$ in spaces of functions defined on this set. By $(\cdot, \cdot),\|\cdot\|$ we will denote respectively the scalar product and the norm in $L^{2}$. We will also use the notation $\mathcal{E}_{0}=H_{0}^{1} \times L^{2}$ for the energy space. Its norm is defined by $\|(u, v)\|_{\mathcal{E}_{0}}^{2}=\|\nabla u\|^{2}+\|v\|^{2}$. Throughout this paper, we denote a generic positive constant by $C$, which values can vary from on line to another. We recall some useful information concerning the spectral fractional Laplacian [1]. Denote by $\left\{e_{i}\right\}_{i=1}^{\infty}$ the eigenfunctions (unitary in $L^{2}(\Omega)$ ) of the operator $-\Delta$ with the Dirichlet boundary conditions, such that the corresponding eigenvalues are given by

$$
0<\lambda_{1} \leq \lambda_{2} \leq \ldots \leq \lambda_{n} \leq \ldots
$$

For $u \in L^{2}$ its $k$-th Fourier coefficient is defined as $\widehat{u}_{k}=\left(u, e_{k}\right)$. Let $s \geq 0$. The spectral fractional Laplacian is defined by the formula

$$
(-\Delta)^{\frac{s}{2}} u=\sum_{k=1}^{\infty} \lambda_{k}^{\frac{s}{2}} \widehat{u}_{k} .
$$


The space $\mathbb{H}^{s}$ is defined as

$$
\mathbb{H}^{s}=\left\{u \in L^{2}: \sum_{k=1}^{\infty} \lambda_{k}^{s} \widehat{u}_{k}^{2}<\infty\right\}
$$

The corresponding norm is given by

$$
\|u\|_{\mathbb{H}^{s}}=\left\|(-\Delta)^{s / 2} u\right\|=\sqrt{\sum_{k=1}^{\infty} \lambda_{k}^{s} \widehat{u}_{k}^{2}}
$$

The space $\mathbb{H}^{s}$ is a subspace of the fractional Sobolev space $H^{s}$. In particular

$$
\mathbb{H}^{s}=\left\{\begin{array}{l}
H^{s}=H_{0}^{s} \text { for } s \in(0,1 / 2) \\
H_{0}^{s} \text { for } s \in(1 / 2,1]
\end{array}\right.
$$

We also remind that the standard fractional Sobolev norm satisfies $\|u\|_{H^{s}} \leq C\|u\|_{\mathbb{H}^{s}}$ for $u \in \mathbb{H}^{s}$, cf. [1, Proposition 2.1]. For $s \in[0,1]$ we will use the notation $\mathcal{E}_{s}=$ $\mathbb{H}^{s+1} \times \mathbb{H}^{s}$. This space is equipped with the norm $\|(u, v)\|_{\mathcal{E}_{s}}^{2}=\|u\|_{\mathbb{H}^{s+1}}^{2}+\|v\|_{\mathbb{H}^{s}}^{2}$.

\section{Problem Definition and Assumptions}

We consider the following family of problems parameterized by $\varepsilon>0$

$$
\left\{\begin{array}{l}
u_{t t}+u_{t}-\Delta u=f_{\varepsilon}(t, u) \text { for }(x, t) \in \Omega \times(0, \infty) \\
u(t, x)=0 \text { for } x \in \partial \Omega \\
u(0, x)=u_{0}(x) \\
u_{t}(0, x)=u_{1}(x)
\end{array}\right.
$$

The initial data has the regularity $\left(u_{0}, u_{1}\right) \in \mathcal{E}_{0}$. Throughout the article we always assume that the non-autonomous and nonlinear term $f_{\varepsilon}(t, u)$, treated as the mapping which assigns to the time $t \in \mathbb{R}$ the function of the variable $u$, belongs to the space $C\left(\mathbb{R} ; C^{1}(\mathbb{R})\right)$. This space is equipped with the metric

$$
\begin{aligned}
& d_{C\left(\mathbb{R} ; C^{1}(\mathbb{R})\right)}\left(g_{1}, g_{2}\right) \\
& \quad=\sum_{i=1}^{\infty} \frac{1}{2^{i}} \frac{\sup _{t \in[-i, i]} d_{C^{1}(\mathbb{R})}\left(g_{1}(t, .), g_{2}(t, .)\right)}{1+\sup _{t \in[-i, i]} d_{C^{1}(\mathbb{R})}\left(g_{1}(t, .), g_{2}(t, .)\right)} \text { for } g_{1}, g_{2} \in C\left(\mathbb{R} ; C^{1}(\mathbb{R})\right),
\end{aligned}
$$

where the metric in $C^{1}(\mathbb{R})$ is defined as follows

$$
d_{C^{1}(\mathbb{R})}\left(g_{1}, g_{2}\right)=\sum_{i=1}^{\infty} \frac{1}{2^{i}} \frac{\left\|g_{1}(u)-g_{2}(u)\right\|_{C^{1}([-i, i])}}{1+\left\|g_{1}(u)-g_{2}(u)\right\|_{C^{1}([-i, i])}} \text { for } g_{1}, g_{2} \in C^{1}(\mathbb{R}),
$$


and $\|g\|_{C^{1}(A)}=\max _{r \in A}|g(r)|+\max _{r \in A}\left|g^{\prime}(r)\right|$ for a compact set $A \subset \mathbb{R}$.

Remark 3.1 If $g_{n} \rightarrow g$ in $C\left(\mathbb{R} ; C^{1}(\mathbb{R})\right)$ then $g_{n} \rightarrow g$ and $\frac{\partial g_{n}}{\partial u} \rightarrow \frac{\partial g}{\partial u}$ uniformly on every bounded subset of $\mathbb{R}$.

We make the following assumptions on functions $f_{\varepsilon}: \mathbb{R} \times \mathbb{R} \rightarrow \mathbb{R}$ and $f_{0}: \mathbb{R} \rightarrow \mathbb{R}$

(H1) For every $\varepsilon \in(0,1]$ the function $f_{\varepsilon} \in C\left(\mathbb{R} ; C^{1}(\mathbb{R})\right)$, and $f_{0} \in C^{1}(\mathbb{R})$.

(H2) For every $u \in \mathbb{R}$

$$
\lim _{\varepsilon \rightarrow 0} \sup _{t \in \mathbb{R}}\left|f_{\varepsilon}(t, u)-f_{0}(u)\right|=0 .
$$

(H3) The following holds

$$
\sup _{\varepsilon \in[0,1]} \sup _{t \in \mathbb{R}} \sup _{u \in \mathbb{R}}\left|f_{\varepsilon}(t, u)-f_{0}(u)\right|<\infty \text {. }
$$

(H4) The following holds

$$
\limsup _{|u| \rightarrow \infty} \frac{f_{0}(u)}{u}<\lambda_{1}
$$

where $\lambda_{1}$ is the first eigenvalue of $-\Delta$ operator with the Dirichlet boundary conditions.

(H5) There exist $0<\kappa \leq 4$ and $C>0$ such that

$$
\sup _{\varepsilon \in[0,1]} \sup _{t \in \mathbb{R}}\left|\frac{\partial f_{\varepsilon}}{\partial u}(t, u)\right| \leq C\left(1+|u|^{4-\kappa}\right) \text { for every } u \in \mathbb{R} .
$$

(H6) For any fixed $u \in \mathbb{R}$ the map $f_{\varepsilon}(t, u)$ is uniformly continuous with respect to $t$. Moreover for every $R>0$ the map $\mathbb{R} \times[-R, R] \ni(t, u) \mapsto \frac{\partial f_{\varepsilon}}{\partial u}(t, u)$ is uniformly continuous.

Remark 3.2 An example of family of functions satisfying conditions (H1)-(H6) is $f_{\varepsilon}(t, u)=-u|u|^{4-\kappa}+g(u)+\varepsilon \sin (t) \sin \left(u^{3}\right)$ where the growth of $g(u)$ is essentially lower than $5-\kappa$.

Proposition 3.3 Assuming (H1), (H5), and (H6), for every $\varepsilon \in[0,1]$ and every $R>0$ the mapping

$$
\mathbb{R} \times[-R, R] \ni(t, u) \mapsto f_{\varepsilon}(t, u)
$$

is uniformly continuous.

Proof Let $u_{1}, u_{2} \in[-R, R]$ and $t_{1}, t_{2} \in \mathbb{R}$. Using (H5), the following holds

$$
\begin{aligned}
& \left|f_{\varepsilon}\left(t_{1}, u_{1}\right)-f_{\varepsilon}\left(t_{2}, u_{2}\right)\right| \leq\left|f_{\varepsilon}\left(t_{1}, u_{1}\right)-f_{\varepsilon}\left(t_{1}, u_{2}\right)\right|+\left|f_{\varepsilon}\left(t_{1}, u_{2}\right)-f_{\varepsilon}\left(t_{2}, u_{2}\right)\right| \\
& \quad \leq C\left(1+R^{4-\kappa}\right)\left|u_{1}-u_{2}\right|+\sup _{|u| \leq R}\left|f_{\varepsilon}\left(t_{1}, u\right)-f_{\varepsilon}\left(t_{2}, u\right)\right| .
\end{aligned}
$$


It suffices to prove that for every $\eta>0$ we can find $\delta>0$ such that if only $\left|t_{1}-t_{2}\right| \leq \delta$ then $\sup _{|u| \leq R}\left|f_{\varepsilon}\left(t_{1}, u\right)-f_{\varepsilon}\left(t_{2}, u\right)\right| \leq \eta$. Assume for contradiction that there exists $\eta_{0}>0$ such that for every $n \in \mathbb{N}$ we can find $t_{1}^{n}, t_{2}^{n} \in \mathbb{R}$ with $\left|t_{1}-t_{2}\right| \leq \frac{1}{n}$ and

$$
\sup _{|u| \leq R}\left|f_{\varepsilon}\left(t_{1}^{n}, u\right)-f_{\varepsilon}\left(t_{2}^{n}, u\right)\right|>\eta_{0}
$$

For every $n$ there exists $u^{n}$ with $\left|u^{n}\right| \leq R$ such that

$$
\left|f_{\varepsilon}\left(t_{1}^{n}, u^{n}\right)-f_{\varepsilon}\left(t_{2}^{n}, u^{n}\right)\right|>\eta_{0}
$$

For a subsequence $u^{n} \rightarrow u^{0}$ with $\left|u^{0}\right| \leq R$, we have

$$
\begin{aligned}
\eta_{0} & <\left|f_{\varepsilon}\left(t_{1}^{n}, u^{n}\right)-f_{\varepsilon}\left(t_{1}^{n}, u^{0}\right)\right|+\left|f_{\varepsilon}\left(t_{1}^{n}, u^{0}\right)-f_{\varepsilon}\left(t_{2}^{n}, u^{0}\right)\right|+\left|f_{\varepsilon}\left(t_{2}^{n}, u^{0}\right)-f_{\varepsilon}\left(t_{2}^{n}, u^{n}\right)\right| \\
& \leq 2 C\left(1+R^{4-\kappa}\right)\left|u^{n}-u^{0}\right|+\left|f_{\varepsilon}\left(t_{1}^{n}, u^{0}\right)-f_{\varepsilon}\left(t_{2}^{n}, u^{0}\right)\right|
\end{aligned}
$$

where in the last estimate we used (H5). By taking $n$ large enough we deduce that

$$
\frac{\eta_{0}}{2}<\left|f_{\varepsilon}\left(t_{1}^{n}, u^{0}\right)-f_{\varepsilon}\left(t_{2}^{n}, u^{0}\right)\right|
$$

a contradiction with uniform continuity of $f_{\varepsilon}\left(\cdot, u_{0}\right)$ assumed in (H6).

We define hull of $f$ as the set $\mathcal{H}(f):=\overline{\left\{f(t+\cdot, \cdot) \in C\left(\mathbb{R} ; C^{1}(\mathbb{R})\right)\right\}_{t \in \mathbb{R}}}$, where the closure is understood in the metric $d_{C\left(\mathbb{R} ; C^{1}(\mathbb{R})\right)}$. We also define set

$$
\mathcal{H}_{[0,1]}:=\bigcup_{\varepsilon \in[0,1]} \mathcal{H}\left(f_{\varepsilon}\right)=\bigcup_{\varepsilon \in(0,1]} \mathcal{H}\left(f_{\varepsilon}\right) \cup\left\{f_{0}\right\},
$$

where the last equality follows from the simple fact that $\mathcal{H}\left(f_{0}\right)=\left\{f_{0}\right\}$. We say that a function $f$ is translation compact if its hull $\mathcal{H}(f)$ is a compact set. The following characterization of translation compactness can be found in [15, Proposition 2.5 and Remark 2.2].

Proposition 3.4 Let $f \in C\left(\mathbb{R} ; C^{1}(\mathbb{R})\right)$. Then $f$ is translation compact if and only if for every $R>0$

(i) $|f(t, u)|+\left|\frac{\partial f}{\partial u}(t, u)\right| \leq C_{R}$ for $(t, u) \in \mathbb{R} \times[-R, R]$,

(ii) The functions $f(t, u)$ and $\frac{\partial f}{\partial u}(t, u)$ are uniformly continuous on $\mathbb{R} \times[-R, R]$.

We prove two simple results concerning the translation compactness of $f_{\varepsilon}$ and each function in its hull.

Corollary 3.5 Assuming (H1), (H3), (H5), and (H6) for every $\varepsilon \in(0,1]$ function $f_{\varepsilon}$ is translation compact.

Proof From assumption (H3) and the fact that $f_{0} \in C^{1}(\mathbb{R})$ one can deduce that (i) from Proposition 3.4 holds. Moreover, (H6) and Proposition 3.3 imply that (ii) holds, and the proof is complete. 
Proposition 3.6 If $f_{\varepsilon}$ satisfies conditions (H1), (H2), (H3), and (H5) then these conditions are satisfied by all elements from $\mathcal{H}_{[0,1]}$. Moreover there exist constants $C, K>0$ independent of $\varepsilon$ such that for every $p_{\varepsilon} \in \mathcal{H}\left(f_{\varepsilon}\right)$ the following bounds hold

$$
\begin{aligned}
& \sup _{\epsilon \in[0,1]} \sup _{t \in \mathbb{R}} \sup _{u \in \mathbb{R}}\left|p_{\varepsilon}(t, s)-f_{0}(u)\right| \\
& \leq K, \quad \sup _{\varepsilon \in[0,1]} \sup _{t \in \mathbb{R}}\left|\frac{\partial p_{\varepsilon}}{\partial u}(t, u)\right| \leq C\left(1+|u|^{4-\kappa}\right) \text { for every } u \in \mathbb{R} .
\end{aligned}
$$

Proof Property (H1) is clear. Suppose that $(\mathrm{H} 2)$ does not hold. Then there exists a number $\delta>0$, sequences $\varepsilon_{n} \rightarrow 0, p_{\varepsilon_{n}} \in \mathcal{H}\left(f_{\varepsilon}\right), t_{n} \in \mathbb{R}$ and a number $u \in \mathbb{R}$ such that

$$
\left|p_{\varepsilon_{n}}\left(t_{n}, u\right)-f_{0}(u)\right|>2 \delta
$$

Because $p_{\varepsilon_{n}} \in \mathcal{H}\left(f_{\varepsilon_{n}}\right)$ we can pick a sequence $s_{n}$ such that $\mid f_{\varepsilon_{n}}\left(s_{n}+t_{n}, u\right)-$ $p_{\varepsilon_{n}}\left(t_{n}, u\right) \mid \leq \delta$. Then

$$
\left|f_{\varepsilon_{n}}\left(t_{n}+s_{n}, u\right)-f_{0}(u)\right| \geq-\left|f_{\varepsilon_{n}}\left(t_{n}+s_{n}, u\right)-p_{\varepsilon_{n}}\left(t_{n}, u\right)\right|+\left|p_{\varepsilon_{n}}\left(t_{n}, u\right)-f_{0}(u)\right| \geq \delta .
$$

Now (H2) follows by contradiction. We denote

$$
K:=\sup _{\varepsilon \in[0,1]} \sup _{t \in \mathbb{R}} \sup _{u \in \mathbb{R}}\left|f_{\varepsilon}(t, u)-f_{0}(u)\right|
$$

which from assumption $(\mathrm{H} 3)$ is a finite number. Taking $p_{\varepsilon} \in \mathcal{H}_{[0,1]}$, for every $(t, u) \in$ $\mathbb{R}^{2}$ we obtain

$$
\begin{aligned}
& \left|p_{\varepsilon}(t, u)-f_{0}(u)\right| \leq\left|f_{\varepsilon}\left(t+s_{n}, u\right)-p_{\varepsilon}(t, u)\right| \\
& \quad+\left|f_{\varepsilon}\left(t+s_{n}, u\right)-f_{0}(u)\right| \leq K+\left|f_{\varepsilon}\left(t+s_{n}, u\right)-p_{\varepsilon}(t, u)\right| .
\end{aligned}
$$

We can pick a sequence $s_{n}$ such that $\left|f_{\varepsilon}\left(s_{n}+t, u\right)-p_{\varepsilon}(t, u)\right| \rightarrow 0$. So, passing to the limit, we get

$$
\left|p_{\varepsilon}(t, u)-f_{0}(u)\right| \leq K .
$$

We have proved that for every $p_{\varepsilon} \in \mathcal{H}_{[0,1]}$ we have

$$
\sup _{t \in \mathbb{R}} \sup _{u \in \mathbb{R}}\left|p_{\varepsilon}(t, u)-f_{0}(u)\right| \leq K .
$$

From (H5) we obtain

$$
\left|\frac{\partial f_{\varepsilon}}{\partial u}\left(t+s_{n}, u\right)\right| \leq C\left(1+|u|^{4-\kappa}\right),
$$


for every $u, t, s_{n} \in \mathbb{R}, \varepsilon \in[0,1]$. Again by choosing $s_{n}$ such that $\mid \frac{\partial f_{\varepsilon}}{\partial u}\left(s_{n}+t, u\right)-$ $\frac{\partial p_{\varepsilon}}{\partial u}(t, u) \mid \rightarrow 0$ and passing to the limit we observe that for every $p_{\varepsilon} \in \mathcal{H}_{[0,1]}$ the following holds

$$
\sup _{t \in \mathbb{R}}\left|\frac{\partial p_{\varepsilon}}{\partial u}(t, u)\right| \leq C\left(1+|u|^{4-\kappa}\right) \text { for every } u \in \mathbb{R}
$$

which completes the proof.

Proposition 3.7 If (H1), (H2), (H3), and (H5) hold, then for every $R>0$ and every $p_{\varepsilon} \in \mathcal{H}\left(f_{\varepsilon}\right)$

$$
\lim _{\varepsilon \rightarrow 0} \sup _{|u| \leq R} \sup _{t \in \mathbb{R}}\left|p_{\varepsilon}(t, u)-f_{0}(u)\right|=0
$$

Proof By contradiction, assume that there exist $\delta>0$ and sequences $\left|u_{n}\right| \leq R, t_{n} \in \mathbb{R}$, $\varepsilon_{n} \rightarrow 0$ such that

$$
\delta \leq\left|p_{\varepsilon_{n}}\left(t_{n}, u_{n}\right)-f_{0}\left(u_{n}\right)\right|
$$

Passing to a subsequence, if necessary, we can assume that $u_{n} \rightarrow u_{0}$, where $\left|u_{0}\right| \leq R$. Hence,

$$
\begin{aligned}
\delta & \leq\left|p_{\varepsilon_{n}}\left(t_{n}, u_{n}\right)-p_{\varepsilon_{n}}\left(t_{n}, u_{0}\right)\right|+\left|p_{\varepsilon_{n}}\left(t_{n}, u_{0}\right)-f_{0}\left(u_{0}\right)\right|+\left|f_{0}\left(u_{0}\right)-f_{0}\left(u_{n}\right)\right| \\
& \leq C\left(1+\left|\xi_{n}\right|^{4-\kappa}\right)\left|u_{n}-u_{0}\right|+\sup _{t \in \mathbb{R}}\left|p_{\varepsilon_{n}}\left(t, u_{0}\right)-f_{0}\left(u_{0}\right)\right|+\left|f_{0}\left(u_{0}\right)-f_{0}\left(u_{n}\right)\right| \\
& \leq C\left(1+|R|^{4-\kappa}\right)\left|u_{n}-u_{0}\right|+\sup _{t \in \mathbb{R}}\left|p_{\varepsilon_{n}}\left(t, u_{0}\right)-f_{0}\left(u_{0}\right)\right|+\left|f_{0}\left(u_{0}\right)-f_{0}\left(u_{n}\right)\right|,
\end{aligned}
$$

where $\xi_{n}$ is an intermediate point between $u_{0}$ and $u_{n}$, where $\xi_{n}$ is a point between $s_{n}$ and $s_{0}$ (which is (H5) applied to $p_{\varepsilon_{n}}$ ).

\section{Galerkin Solutions}

Definition 4.1 Let $\left(u_{0}, u_{1}\right) \in \mathcal{E}_{0}$. The function $u \in L_{l o c}^{\infty}\left([0, \infty) ; H_{0}^{1}\right)$ with $u_{t} \in$ $L_{l o c}^{\infty}\left([0, \infty) ; L^{2}\right)$ and $u_{t t} \in L_{l o c}^{\infty}\left([0, \infty) ; H^{-1}\right)$ is a weak solution of problem (3.1) if for every $v \in L_{l o c}^{2}\left([0, \infty) ; H_{0}^{1}\right)$ and $t_{1}>0$ the following holds

$$
\int_{0}^{t_{1}}\left\langle u_{t t}(t), v(t)\right\rangle_{H^{-1} \times H_{0}^{1}}+\left(u_{t}(t)-f_{\varepsilon}(t, u(t)), v\right)+(\nabla u(t), \nabla v(t)) d t=0,
$$

and $u(0)=u_{0}, u_{t}(0)=u_{1}$.

Note that as $u \in C\left([0, \infty) ; L^{2}\right)$ and $u_{t} \in C\left([0, \infty) ; H^{-1}\right)$, pointwise values of $u$ and $u_{t}$, and thus the initial data, make sense. However, due to the lack of regularity of the 
nonlinear term $f_{\varepsilon}(\cdot, u(\cdot))$, we cannot test the equation with $u_{t}$. Indeed, by the Sobolev embedding $H_{0}^{1} \hookrightarrow L^{6}$, it holds that $u(t) \in L^{6}$ for a.e. $t$ and by (H5) for the expression

$$
\int_{\Omega} f_{\varepsilon}(t, u(x, t)) u_{t}(x, t) d x
$$

to make sense we would need $u \in L^{10-2 \kappa}$, which we cannot guarantee. Thus, although it is straightforward to prove (using the Galerkin method) the existence of the weak solution given by the above definition, we cannot establish the energy estimates required to work with this solution.

Let $\left\{e_{i}\right\}_{i=1}^{\infty}$ be the eigenfunctions of the $-\Delta$ operator with the Dirichlet boundary conditions on $\partial \Omega$ sorted by the nondecreasing eigenvalues. They constitute an orthonormal basis of $L^{2}$ and they are orthogonal in $H_{0}^{1}$. Denote $V_{N}=$ span $\left\{e_{1}, \ldots, e_{N}\right\}$. The family of finite dimensional spaces $\left\{V_{N}\right\}_{N=1}^{\infty}$ approximates $H_{0}^{1}$ from the inside, that is

$$
\overline{\bigcup_{N=1}^{\infty} V_{N}} H_{0}^{1}=H_{0}^{1} \quad \text { and } \quad V_{N} \subset V_{N+1} \text { for every } N \geq 1
$$

Let $u_{0}^{N} \in V_{N}$ and $u_{1}^{N} \in V_{N}$ be such that

$$
\begin{aligned}
& u_{0}^{N} \rightarrow u_{0} \text { in } H_{0}^{1} \text { as } N \rightarrow \infty \\
& u_{1}^{N} \rightarrow u_{1} \text { in } L^{2} \text { as } N \rightarrow \infty .
\end{aligned}
$$

Now the $N$-th Galerkin approximate solution for (3.1) is defined as follows.

Definition 4.2 The function $u^{N} \in C^{1}\left([0, \infty) ; V_{N}\right)$ with $u_{t}^{N} \in A C\left([0, \infty) ; V_{N}\right)$ is the $N$-th Galerkin approximate solution of problem (3.1) if $u^{N}(0)=u_{0}^{N}, u_{t}^{N}(0)=u_{1}^{N}$ and for every $v \in V_{N}$ and a.e. $t>0$ the following hold

$$
\left(u_{t t}^{N}(t)+u_{t}^{N}(t)-f_{\varepsilon}\left(t, u^{N}(t)\right), v\right)+\left(\nabla u^{N}(t), \nabla v\right)=0 .
$$

We continue by defining the weak solution of Galerkin type.

Definition 4.3 The weak solution given by Definition 4.1 is said to be of Galerkin type if it is a limit of the subsequence of solutions of the Galerkin problems, understood in the following sense

$$
\begin{aligned}
& u^{N} \rightarrow u \text { weakly-* in } L_{l o c}^{\infty}\left([0, \infty) ; H_{0}^{1}\right), \\
& u_{t}^{N} \rightarrow u_{t} \text { weakly-* in } L_{l o c}^{\infty}\left([0, \infty) ; L^{2}\right), \\
& u_{t t}^{N} \rightarrow u_{t t} \text { weakly-* in } L_{l o c}^{\infty}\left([0, \infty) ; H^{-1}\right) .
\end{aligned}
$$

For brevity of notation we use the index $N$ to denote the elements of the subsequence. 
In the sequel we will consider problem (3.1) with $p \in \mathcal{H}_{[0,1]}$ replacing $f_{\varepsilon}$. In particular, the next two results hold for the function $f_{\varepsilon}$ in (3.1) replaced by $p \in \mathcal{H}_{[0,1]}$. We skip the proof of the following theorem which is standard in the framework of the Galerkin method.

Theorem 4.4 Assume (H1), (H3)-(H5). If $\left(u_{0}, u_{1}\right) \in \mathcal{E}_{0}$ then then problem (3.1) has at least one weak solution of Galerkin type.

Proposition 4.5 The weak solutions of Galerin type of problem (3.1) are bounded in $\mathcal{E}_{0}$ and there exists a bounded set $B_{0} \subset \mathcal{E}_{0}$ which is absorbing, i.e. for every bounded set $B \subset \mathcal{E}_{0}$ there exists $t_{0} \geq 0$ such that for every weak solution of Galerkin type $\left(u(t), u_{t}(t)\right)$ with the initial conditions in $B$ we have $\left(u(t), u_{t}(t)\right) \in B_{0}$ for every $t \geq t_{0}$. Moreover $B_{0}$ and $t_{1}$ do not depend on the choice of $p \in \mathcal{H}_{[0,1]}$ in place of $f_{\varepsilon}$ in (3.1).

To prove the above proposition we will need the following Gronwall type lemma.

Lemma 4.6 Let $I:[0, \infty) \rightarrow \mathbb{R}$ be an absolutely continuous function with $I(t)=$ $\sum_{i=1}^{k} I_{i}(t)$. Suppose that

$$
\frac{d}{d t} I(t) \leq-A_{i} I_{i}(t)^{\alpha_{i}}+B_{i}
$$

for every $i \in\{1, \ldots, k\}$ and for almost every $t$ such that $I_{i}(t) \geq 0$, where $\alpha_{i}, A_{i}, B_{i}>$ 0 are constants. Then for every $\eta>0$ there exists $t_{0}>0$ such that

$$
I(t) \leq \sum_{i=1}^{k}\left(\frac{B_{i}}{A_{i}}\right)^{\frac{1}{\alpha_{i}}}+\eta, \text { for every } t \geq t_{0} .
$$

If, in addition, $\left\{I^{l}(t)\right\}_{l \in \mathcal{L}}$ is a family of functions satisfying the above conditions and such that $I^{l}(0) \leq Q$ for each $l \in \mathcal{L}$, then the time $t_{0}$ is independent of $l$ and there exists a constant $C$ depending on $Q, A_{i}, B_{i}, \alpha_{i}$ such that $I^{l}(t) \leq C$ for every $t \geq 0$ and every $l \in \mathcal{L}$.

Proof We denote

$$
B=\sum_{i=1}^{k}\left(\frac{B_{i}}{A_{i}}\right)^{\frac{1}{\alpha_{i}}}
$$

and let $A=\min _{i \in\{1, \ldots, k\}}\left\{A_{i}\right\}$. First we will show that for every $\eta>0$ if $I\left(t_{0}\right) \leq B+\eta$, then $I(t) \leq B+\eta$ for every $t \geq t_{0}$. For the sake of contradiction let us suppose that there exists some $t_{1}>t_{0}$ such that $I\left(t_{1}\right)>B+\eta$. Let $t_{2}=\sup \left\{s \in\left[t_{0}, t_{1}\right]: I(s) \leq B+\eta\right\}$. Choose $\delta>0$ such that

$$
\eta \geq \sum_{i=1}^{k}\left(\left(\frac{B_{i}}{A_{i}}+\delta\right)^{\frac{1}{\alpha_{i}}}-\left(\frac{B_{i}}{A_{i}}\right)^{\frac{1}{\alpha_{i}}}\right) .
$$


Hence

$$
I(s)>B+\eta \geq \sum_{i=1}^{k}\left(\frac{B_{i}}{A_{i}}+\delta\right)^{\frac{1}{\alpha_{i}}} \quad \text { for } s \in\left(t_{2}, t_{1}\right]
$$

We deduce that for every $s \in\left(t_{2}, t_{1}\right]$ we can find an index $i$ (which may depend on $s$ ) for which

$$
I_{i}(s)>\left(\frac{B_{i}}{A_{i}}+\delta\right)^{\frac{1}{\alpha_{i}}}
$$

Then for a.e. $s \in\left(t_{2}, t_{1}\right]$ we have

$$
\frac{d}{d t} I(s) \leq-A_{i} I_{i}(s)^{\alpha_{i}}+B_{i} \leq-A \delta
$$

and after integration we get that $I\left(t_{1}\right)<I\left(t_{2}\right)-\left(t_{2}-t_{1}\right) A \delta$ which is a contradiction. We observe that all functions from the family $\left\{I^{l}(t)\right\}_{l \in \mathcal{L}}$ are bounded by $\max \{Q, 1\}+B$. Now we will prove existence of $t_{0}$. For the sake of contradiction suppose that there exists $\eta>0$ and the sequence of times $t_{n} \rightarrow \infty$ such that $I^{l_{n}}\left(t_{n}\right)>B+\eta$ for some $l_{n} \in \mathcal{L}$. Then for every $s \in\left[0, t_{n}\right]$ we must have $I^{l_{n}}(s)>B+\eta$. Thus, there exists $\delta>0$ such that for all $s \in\left[0, t_{n}\right]$ and $l_{n}$ there exists an index $i \in\{1, \ldots, k\}$ (which may depend on $s$ and $n$ ) for which $I_{i}^{l_{n}}(s)>\left(\frac{B_{i}}{A_{i}}+\delta\right)^{\frac{1}{\alpha_{i}}}$. Again for a.e $s \in\left(0, t_{n}\right)$

$$
\frac{d}{d t} I^{l_{n}}(s) \leq-A \delta
$$

and after integrating we obtain $I^{l_{n}}\left(t_{n}\right) \leq Q-t_{n} A \delta$ for each $n$, which is a contradiction.

Proof of Proposition 4.5 Let $u$ be the Galerkin solution to (3.1) with any function $p \in$ $\mathcal{H}_{[0,1]}$ in place of $f_{\varepsilon}$ at the right-hand side of (3.1). The next estimates hold for the Galerkin problems, but since they do not depend on the dimension of the space used in those problems, they are also satisfied by the limit solution. To make the presentation simpler, we proceed in formal way. By testing the equation with $u+2 u_{t}$ we obtain

$$
\begin{aligned}
& \frac{d}{d t}\left[\left(u_{t}, u\right)+\frac{1}{2}\|u\|^{2}+\left\|u_{t}\right\|^{2}+\|\nabla u\|^{2}-2 \int_{\Omega} F_{0}(u) d x\right] \\
& \quad=-\left\|u_{t}\right\|^{2}-\|\nabla u\|^{2}+\left(f_{0}(u), u\right)+\left(p(t, u)-f_{0}(u), 2 u_{t}+u\right)
\end{aligned}
$$

where $F_{0}(u)=\int_{0}^{u} f_{0}(v) d v$. Assumption (H4) implies the inequality

$$
\left(f_{0}(u), u\right) \leq C+K\|u\|^{2}, \text { where } 0 \leq K<\lambda_{1} .
$$


We define

$$
I(t)=\left(u_{t}, u\right)+\frac{1}{2}\|u\|^{2}+\left\|u_{t}\right\|^{2}+\|\nabla u\|^{2}-2 \int_{\Omega} F_{0}(v) d x .
$$

Using the Poincaré and Cauchy inequalities we obtain

$$
\frac{d}{d t} I(t) \leq-\left\|u_{t}\right\|^{2}-C\|\nabla u\|^{2}+\left\|p(t, u)-f_{0}(u)\right\|\left(2\left\|u_{t}\right\|+\|u\|\right)+C .
$$

Using the Poincaré and Cauchy inequalities again it follows by Proposition 3.6 that

$$
\frac{d}{d t} I(t) \leq-C\left(\left\|u_{t}\right\|^{2}+\|\nabla u\|^{2}\right)+C .
$$

We represent the function $I(t)$ as the sum of the following terms

$$
I_{1}=\left\|u_{t}\right\|^{2}, I_{2}=\frac{1}{2}\|u\|^{2}, I_{3}=\|\nabla u\|^{2}, I_{4}(t)=\left(u_{t}, u\right), I_{5}=-2 \int_{\Omega} F_{0}(u) d x .
$$

From the estimate (4.5) and Poincaré inequality we can easily see that

$$
\frac{d}{d t} I \leq-A_{i} I_{i}+B_{i} \text { for } i \in\{1,2,3,4\}
$$

where $A_{i}, B_{i}$ are positive constants. To deal with the term $I_{5}$ we observe that by the growth condition (H5) using the Hölder inequality we obtain

$$
\begin{aligned}
I_{5} & \leq\left. C \int_{\Omega}\left|\int_{0}^{u} 1+\right| v\right|^{5} d v \mid d x \leq C \int_{\Omega}\left(|u|+|u|^{6}\right) d x \\
& =C\left(\|u\|_{L^{1}}+\|u\|_{L^{6}}^{6}\right) \leq C\left(\|u\|_{L^{6}}+\|u\|_{L^{6}}^{6}\right) \\
& \leq C\left(\|u\|_{L^{6}}^{6}+1\right) .
\end{aligned}
$$

From the Sobolev embedding $H_{0}^{1} \hookrightarrow L^{6}$ it follows that

$$
I_{5}^{\frac{1}{3}} \leq C\left(\|\nabla u\|^{6}+1\right)^{\frac{1}{3}} \leq C\left(\|\nabla u\|^{2}+1\right) .
$$

From the estimate (4.5) we observe that

$$
\frac{d}{d t} I \leq-A_{5} I_{5}^{\frac{1}{3}}+B_{5} \text {, with } A_{5}, B_{5}>0 .
$$

By Lemma 4.6 we deduce that there exists a constant $D>0$ such that every for bounded set of initial data $B \subset \mathcal{E}_{0}$ there exists the time $t_{0}=t_{0}(B)$ such that for every 
$p \in \mathcal{H}_{[0,1]}$ the following holds

$$
I(t) \leq D \text { for } t \geq t_{0} \text { and }\left(u_{0}, u_{1}\right) \in B
$$

We observe that from $(\mathrm{H} 4)$ it follows that

$$
F_{0}(u) \leq C+\frac{K}{2} u^{2} \text { where } 0 \leq K<\lambda_{1}
$$

We deduce

$$
I(t) \geq \frac{1}{2}\left\|u_{t}\right\|^{2}+\|\nabla u\|^{2}-K\|u\|^{2}-C \geq C\|u\|_{\mathcal{E}_{0}}^{2}-C .
$$

We have shown the existence of the absorbing set $B_{0} \subset \mathcal{E}_{0}$ which is independent of the choice of $p \in \mathcal{H}_{[0,1]}$. By Lemma 4.6 it follows that for every initial condition $\left(u_{0}, u_{1}\right) \in \mathcal{E}_{0}$ there exists a constant $D=D\left(u_{0}, u_{1}\right)>0$ such that for every $p \in \mathcal{H}_{[0,1]}$ and $t \in \mathbb{R}$ the following holds

$$
I(t) \leq D \text { for } t \in[0, \infty)
$$

The proof is complete.

\section{Shatah-Struwe Solutions, Their Regularity and a Priori Estimates}

\subsection{Auxiliary Linear Problem}

Similar as in [27] we define an auxiliary non-autonomous problem for which we derive a priori estimates both in energy and Strichartz norms.

$$
\left\{\begin{array}{l}
u_{t t}+u_{t}-\Delta u=G(x, t) \text { for }(x, t) \in \Omega \times\left(t_{0}, \infty\right), \\
u(t, x)=0 \text { for } x \in \partial \Omega \\
u\left(t_{0}, x\right)=u_{0}(x) \\
u_{t}\left(t_{0}, x\right)=u_{1}(x)
\end{array}\right.
$$

It is well known that if only $G \in L_{l o c}^{1}\left(\left[t_{0}, \infty\right) ; L^{2}\right)$ and $\left(u_{0}, u_{1}\right) \in \mathcal{E}_{0}$ then the problem (5.1) has the unique weak solution $u$ belonging to $C_{l o c}\left(\left[t_{0}, \infty\right) ; H_{0}^{1}\right)$ with $u_{t} \in C_{l o c}\left(\left[t_{0}, \infty\right) ; L^{2}\right)$ and $u_{t t} \in L_{l o c}^{\infty}\left(\left[t_{0}, \infty\right) ; H^{-1}\right)$. This solution is the limit of the Galerkin approximations in the spaces spanned by the eigenfunctions of $-\Delta$ with homogeneous Dirichlet boundary conditions, and $L^{2}$ projections of $u$ on those spaces coincide with the Galerkin solutions. For details, cf. [4,15,34,41]. The next result appears in [27, Proposition 2.1]. For completeness of our argument we provide the outline of the proof. 
Proposition 5.1 Let $u$ be the weak solution to problem $(5.1)$ on the interval $\left[t_{0}, \infty\right)$ with $G \in L_{l o c}^{1}\left(\left[t_{0}, \infty\right) ; L^{2}\right)$ and initial data $u\left(t_{0}\right)=u_{0}, u_{t}\left(t_{0}\right)=u_{1}$ with $\left(u_{0}, u_{1}\right) \in \mathcal{E}_{0}$. Then the following estimate holds

$$
\left\|\left(u(t), u_{t}(t)\right)\right\|_{\mathcal{E}_{0}} \leq C\left(\left\|\left(u_{0}, u_{1}\right)\right\|_{\mathcal{E}_{0}} e^{-\alpha\left(t-t_{0}\right)}+\int_{t_{0}}^{t} e^{-\alpha(t-s)}\|G(s)\| d s\right)
$$

for every $t \geq t_{0}$, where $C, \alpha$ are positive constants independent of $t, t_{0}, G$ and the initial conditions of (5.1).

Proof Testing (5.1) by $u+2 u_{t}$ we obtain

$$
\frac{d}{d t}\left(\left(u_{t}, u\right)+\frac{1}{2}\|u\|^{2}+\left\|u_{t}\right\|^{2}+\|\nabla u\|^{2}\right)=-\left\|u_{t}\right\|^{2}-\|\nabla u\|^{2}+\left(G(t), u+2 u_{t}\right)
$$

We define $I(t)=\left(u_{t}, u\right)+\frac{1}{2}\|u\|^{2}+\left\|u_{t}\right\|^{2}+\|\nabla u\|^{2}$. We easily deduce

$$
\frac{d}{d t} I(t) \leq C(-I(t)+\sqrt{I(t)}\|G(t)\|)
$$

Multiplying the above inequality by $e^{C t}$ we obtain

$$
\frac{d}{d t}\left(I(t) e^{C t}\right) \leq C e^{C t}\|G(t)\| \sqrt{I(t)}
$$

After integration it follows that

$$
I(t) e^{C t}-I\left(t_{0}\right) e^{C t_{0}} \leq C \int_{t_{0}}^{t} e^{C s}\|G(s)\| \sqrt{I(s)} d s .
$$

Hence, for every $\varepsilon>0$

$$
I(t) \leq\left(I\left(t_{0}\right)+\varepsilon\right) e^{C\left(t_{0}-t\right)}+e^{-C t} C \int_{t_{0}}^{t} e^{C s}\|G(s)\| \sqrt{I(s)} d s
$$

Now let

$$
J(t)=C \int_{t_{0}}^{t} e^{C s}\|G(s)\| \sqrt{I(s)} d s .
$$

Then $J$ is absolutely continuous, $J\left(t_{0}\right)=0$, and for almost every $t>t_{0}$ we obtain

$$
J^{\prime}(t)=C e^{C t}\|G(t)\| \sqrt{I(t)}
$$


From (5.2) it follows that

$$
\begin{aligned}
J^{\prime}(t) & \leq C e^{C t}\|G(t)\| \sqrt{\left(I\left(t_{0}\right)+\varepsilon\right) e^{C\left(t_{0}-t\right)}+e^{-C t} J(t)} \\
& =C e^{\frac{C t}{2}}\|G(t)\| \sqrt{\left(I\left(t_{0}\right)+\varepsilon\right) e^{C t_{0}}+J(t)} .
\end{aligned}
$$

Hence

$$
\frac{J^{\prime}(t)}{\sqrt{\left(I\left(t_{0}\right)+\varepsilon\right) e^{C t_{0}}+J(t)}} \leq C e^{\frac{C t}{2}}\|G(t)\|
$$

which makes sense since the denominator in positive as $\varepsilon>0$. After integrating over interval $\left[t_{0}, t\right]$ we obtain the following inequality valid for every $t \geq t_{0}$

$$
\sqrt{\left(I\left(t_{0}\right)+\varepsilon\right) e^{C t_{0}}+J(t)} \leq \sqrt{\left(I\left(t_{0}\right)+\varepsilon\right) e^{C t_{0}}}+\frac{C}{2} \int_{t_{0}}^{t} e^{\frac{C s}{2}}\|G(s)\| d s .
$$

It follows that

$$
J(t) \leq C\left[\left(\int_{t_{0}}^{t} e^{\frac{C s}{2}}\|G(s)\| d s\right)^{2}+\left(I\left(t_{0}\right)+\varepsilon\right) e^{C t_{0}}\right] .
$$

From definition of $J(t)$ using the inequality (5.2) we notice that

$$
I(t) \leq C\left(\left(I\left(t_{0}\right)+\varepsilon\right) e^{\alpha\left(t_{0}-t\right)}+\left(\int_{t_{0}}^{t} e^{-\alpha(t-s)}\|G(s)\| d s\right)^{2}\right)
$$

for a constant $\alpha>0$. As $c_{1}\left\|\left(u(t), u_{t}(t)\right)\right\|_{\mathcal{E}_{0}} \leq \sqrt{I(t)} \leq c_{2}\left\|\left(u(t), u_{t}(t)\right)\right\|_{\mathcal{E}_{0}}$ for some $c_{1}, c_{2}>0$, passing with $\varepsilon$ to zero we obtain the required assertion.

The following lemma provides us an extra control on the $L^{4}\left(L^{12}\right)$ norm of the solution to the linear problem (5.1). The result is given in [27, Proposition 2.2 and Remark 2.3].

Lemma 5.2 Let $h>0$ and let $u$ be a weak solution to problem (5.1) on the time interval $\left(t_{0}, t_{0}+h\right)$ with $G \in L^{1}\left(t_{0}, t_{0}+h ; L^{2}\right)$ and $\left(u\left(t_{0}\right), u_{t}\left(t_{0}\right)\right)=\left(u_{0}, u_{1}\right) \in \mathcal{E}_{0}$. Then $u \in L^{4}\left(t_{0}, t_{0}+h ; L^{12}\right)$ and the following estimate holds

$$
\|u\|_{L^{4}\left(t_{0}, t_{0}+h ; L^{12}\right)} \leq C_{h}\left(\left\|\left(u_{0}, u_{1}\right)\right\|_{\mathcal{E}_{0}}+\|G\|_{L^{1}\left(t_{0}, t_{0}+h ; L^{2}\right)}\right),
$$

where the constant $C_{h}>0$ depends only on $h$ but is independent of $t_{0},\left(u_{0}, u_{1}\right), G$.

We will need the following result.

Proposition 5.3 It is possible to choose the constants $C_{h}$ in previous lemma such that the function $[0, \infty) \ni h \rightarrow C_{h}$ is nondecreasing. 
The above proposition will be proved with the use of the following theorem known as the Christ-Kiselev Lemma, see e.g. [38, Lemma 3.1].

Theorem 5.4 Let $X, Y$ be Banach spaces and assume that $K(t, s)$ is a continuous function taking values in $B(X, Y)$, the space of linear bounded mappings from $X$ to $Y$. Suppose that $-\infty \leq a<b \leq \infty$ and set

$$
\begin{aligned}
T f(t) & =\int_{a}^{b} K(t, s) f(s) d s, \\
W f(t) & =\int_{a}^{t} K(t, s) f(s) d s .
\end{aligned}
$$

If, for $1 \leq p<q \leq \infty$ it holds that

$$
\|T f\|_{L^{q}(a, b ; Y)} \leq C\|f\|_{L^{p}(a, b ; X)},
$$

then

$$
\|W f\|_{L^{q}(a, b ; Y)} \leq \bar{C}\|f\|_{L^{p}(a, b ; X)}, \text { with } \bar{C}=2 C \frac{2^{2\left(\frac{1}{q}-\frac{1}{p}\right)}}{1-2^{\frac{1}{q}-\frac{1}{p}}}
$$

Proof of Proposition 5.3 If $G \equiv 0$ then we denote the corresponding constant by $D_{h}$, i.e.

$$
\|u\|_{L^{4}\left(t_{0}, t_{0}+h ; L^{12}\right)} \leq D_{h}\left\|\left(u_{0}, u_{1}\right)\right\|_{\mathcal{E}_{0}} .
$$

Clearly, the function $[0, \infty) \ni h \rightarrow D_{h} \in[0, \infty)$ can be made nondecreasing. We will prove that (5.3) holds with $C_{h}$, a monotone function of $D_{h}$. If the family $\{S(t)\}_{t \in \mathbb{R}}$ of mappings $S(t): \mathcal{E}_{0} \rightarrow \mathcal{E}_{0}$ is the solution group for the linear homogeneous problem (i.e. if $G \equiv 0)$ then we denote $S(t)\left(u_{0}, u_{1}\right)=\left(S_{u}(t)\left(u_{0}, u_{1}\right), S_{u_{t}}(t)\left(u_{0}, u_{1}\right)\right)$. Let $t_{0} \in \mathbb{R}$ and $\delta>0$. Using the Duhamel formula for equation (5.1) we obtain

$$
u\left(t_{0}+\delta\right)=S_{u}(\delta)\left(u_{0}, u_{1}\right)+\int_{0}^{\delta} S_{u}(\delta-s)\left(0, G\left(t_{0}+s\right)\right) d s
$$

Applying the $L^{4}\left(0, h ; L^{12}\right)$ norm with respect to $\delta$ to both sides we obtain

$$
\|u\|_{L^{4}\left(t_{0}, t_{0}+h ; L^{12}\right)} \leq D_{h}\left\|\left(u_{0}, u_{1}\right)\right\|_{\mathcal{E}}+\left\|P_{1}\right\|_{L^{4}\left(0, h ; L^{12}\right)}
$$

for every $h>0$, where $P_{1}(\delta)=\int_{0}^{\delta} S_{u}(\delta-s)\left(0, G\left(t_{0}+s\right)\right) d s$. We will estimate the Strichartz norm of $P_{1}$ using Theorem 5.4 with $X=L^{2}, Y=L^{12}, q=4, p=1, a=$ $0, b=h$. If $\Pi_{N}: L^{2} \rightarrow V_{N}$ is $L^{2}$-orthogonal projection, then $S_{u}(h-s)\left(0, \Pi_{N}(\cdot)\right)$ is a continuous function of $(h, s)$ taking its values in $B\left(L^{2}, L^{12}\right)$. Hence the estimate should be derived separately for every $N$, and, since it is uniform with with respect 
to $N$, it holds also in the limit. We skip this technicality and proceed with the formal estimates only. We set $P_{2}(\delta)=\int_{0}^{h} S_{u}(\delta-s)\left(0, G\left(t_{0}+s\right)\right) d s$, and we estimate

$$
\begin{aligned}
\left\|P_{2}\right\|_{L^{4}\left(0, h ; L^{12}\right)} & \leq \int_{0}^{h}\left\|S_{u}(\delta-s)\left(0, G\left(t_{0}+s\right)\right)\right\|_{L^{4}\left(0, h ; L^{12}\right)} d s \\
& =\int_{0}^{h}\left\|S_{u}(\delta) S(-s)\left(0, G\left(t_{0}+s\right)\right)\right\|_{L^{4}\left(0, h ; L^{12}\right)} d s \\
& \leq \int_{0}^{h} D_{h}\left\|S(-s)\left(0, G\left(t_{0}+s\right)\right)\right\|_{\mathcal{E}_{0}} d s,
\end{aligned}
$$

where in the last inequality we used the homogeneous Strichartz estimate. Observe that there exists $\beta>0$ such that

$$
\left\|S(-s)\left(u_{0}, u_{1}\right)\right\|_{\mathcal{E}_{0}} \leq e^{s \beta}\left\|\left(u_{0}, u_{1}\right)\right\|_{\mathcal{E}_{0}}
$$

We deduce

$$
\left\|P_{2}\right\|_{L^{4}\left(0, h ; L^{12}\right)} \leq D_{h} e^{\beta h}\|G\|_{L^{1}\left(t_{0}, t_{0}+h, L^{2}\right)} .
$$

Hence, by Theorem 5.4 we obtain $\left\|P_{1}\right\|_{L^{4}\left(0, h ; L^{12}\right)} \leq C D_{h} e^{\beta h}\|G\|_{L^{1}\left(t_{0}, t_{0}+h, L^{2}\right)}$ for every $h>0$, and the proof is complete.

The following result will be useful in the bootstrap argument on the attractor regularity.

Lemma 5.5 Let $\left(u_{0}, u_{1}\right) \in \mathcal{E}_{s}$ and $G \in L_{\text {loc }}^{1}\left(\left[t_{0}, \infty\right) ; \mathbb{H}^{s}\right)$ for $s \in(0,1]$. Then the weak solution of (5.1) has regularity $u \in C_{\text {loc }}\left(\left[t_{0}, \infty\right) ; \mathbb{H}^{s+1}\right)$ and $u_{t} \in C_{l o c}\left(\left[t_{0}, \infty\right) ; \mathbb{H}^{s}\right)$, Moreover, the following estimates hold

$$
\begin{aligned}
\left\|\left(u(t), u_{t}(t)\right)\right\|_{\mathcal{E}_{s}} & \leq C\left(\left\|\left(u_{0}, u_{1}\right)\right\|_{\mathcal{E}_{s}} e^{-\alpha\left(t-t_{0}\right)}+\int_{t_{0}}^{t} e^{-\alpha(t-s)}\|G(s)\|_{\mathbb{H}^{s}} d s\right), \\
\|u\|_{L^{4}\left(0, h ; W^{s, 12}\right)} & \leq C_{h}\left(\left\|\left(u_{0}, u_{1}\right)\right\|_{\mathcal{E}_{s}}+\|G\|_{L^{1}\left(t_{0}, t_{0}+h ; \mathbb{H}^{s}\right)}\right) .
\end{aligned}
$$

Proof The problem

$$
\left\{\begin{array}{l}
w_{t t}(t)+w_{t}(t)-\Delta w(t)=(-\Delta)^{s / 2} G(t) \text { for }(x, t) \in \Omega \times\left(t_{0}, \infty\right), \\
w(t, x)=0 \text { for } x \in \partial \Omega \\
w\left(t_{0}\right)=(-\Delta)^{s / 2} u_{0} \\
w_{t}\left(t_{0}\right)=(-\Delta)^{s / 2} u_{1}
\end{array}\right.
$$

has the unique weak solution $w \in C_{l o c}\left(\left[t_{0}, \infty\right) ; H_{0}^{1}\right)$ with the derivative $w_{t} \in$ $C_{l o c}\left(\left[t_{0}, \infty\right) ; L^{2}\right)$. Both weak solutions $u$ and $w$ are the limits of the Galerkin problems in the spaces spanned by eigenfunctions of $-\Delta$ with Dirichlet boundary conditions, 
and moreover the $L^{2}$ orthogonal projections of $u$ and $w$ on those spaces coincide with the Galerkin solutions. It is enough to observe that

$$
\widehat{w}_{k}(t)=\lambda_{k}^{\frac{s}{2}} \widehat{u}_{k}(t) \text { for every } k \in \mathbb{N} \text {. }
$$

Testing weak solutions $w, u$ with $e_{k}$, we get systems

$$
\begin{aligned}
& \left\{\begin{array}{l}
\widehat{u}_{k}^{\prime \prime}+\widehat{u}_{k}^{\prime}+\lambda_{k} \widehat{u}_{k}=\left(G(t), e_{k}\right), \\
\widehat{u}_{k}\left(t_{0}\right)=\widehat{u}_{0 k} \\
\widehat{u}_{k}^{\prime}\left(t_{0}\right)=\widehat{u}_{1 k}
\end{array}\right. \\
& \left\{\begin{array}{l}
\widehat{w}_{k}^{\prime \prime}+\widehat{w}_{k}^{\prime}+\lambda_{k} \widehat{w}_{k}=\left((-\Delta)^{\frac{s}{2}} G(t), e_{k}\right)=\lambda_{k}^{\frac{s}{2}}\left(G(t), e_{k}\right), \\
\widehat{w}_{k}\left(t_{0}\right)=\left((-\Delta)^{\frac{s}{2}} u_{0}, e_{k}\right)=\lambda_{k}^{\frac{2}{s}} \widehat{u_{0 k}}, \\
\widehat{w}_{k}^{\prime}\left(t_{0}\right)=\left((-\Delta)^{\frac{s}{2}} u_{1}, e_{k}\right)=\lambda_{k}^{\frac{2}{s}} \widehat{u_{1 k}} .
\end{array}\right.
\end{aligned}
$$

The difference $\bar{w}_{k}(t)=\widehat{w}_{k}(t)-\lambda_{k}^{\frac{s}{2}} \widehat{u}_{k}(t)$ solves the problem

$$
\left\{\begin{array}{l}
\bar{w}_{k}^{\prime \prime}+\bar{w}_{k}^{\prime}+\lambda_{k} \bar{w}_{k}=0, \\
\bar{w}_{k}\left(t_{0}\right)=0 \\
\bar{w}_{k}^{\prime}\left(t_{0}\right)=0
\end{array}\right.
$$

So $\bar{w}_{k}(t)=0$ for every $t \in\left[t_{0}, \infty\right)$. The assertion follows from Proposition 5.1 and Lemma 5.2.

\subsection{Shatah-Struwe Solutions and Their Properties}

This section recollects the results from [27]. The non-autonomous generalizations of these results are straightforward so we skip some of the proofs which follow the lines of the corresponding results from [27]. The following remark follows from the Gagliardo-Nirenberg interpolation inequality and the Sobolev embedding $H_{0}^{1} \hookrightarrow L^{6}$.

Remark 5.6 If $u \in L^{4}\left(0, t ; L^{12}\right)$ and $u \in L^{\infty}\left(0, t ; H_{0}^{1}\right)$ then

$$
\|u\|_{L^{5}\left(0, t ; L^{10}\right)} \leq\|u\|_{L^{4}\left(0, t ; L^{12}\right)}^{\frac{4}{5}}\|u\|_{L^{\infty}\left(0, t ; H_{0}^{1}\right)}^{\frac{1}{5}} .
$$

We define the Shatah-Struwe solution of problem (3.1).

Definition 5.7 Let $\left(u_{0}, u_{1}\right) \in \mathcal{E}_{0}$. A weak solution of problem (3.1), given by Definition 4.1, is called a Shatah-Struwe solution if $u \in L_{l o c}^{4}\left([0, \infty) ; L^{12}\right)$.

Proposition 5.8 Shatah-Struwe solutions to problem (3.1) given by Definition 5.7 are unique and the mapping $\mathcal{E}_{0} \ni\left(u_{0}, u_{1}\right) \mapsto\left(u(t), u_{t}(t)\right) \in \mathcal{E}_{0}$ is continuous for every $t>0$. 
Proof Let $u, v$ be Shatah-Struwe solutions to Problem (3.1) with the initial data $\left(u_{0}, u_{1}\right)$ and $\left(v_{0}, v_{1}\right)$, respectively. Their difference $w:=u-v$ satisfies the following equation

$$
w_{t t}(t)+w_{t}(t)-\Delta w(t)=p(t, u(t))-p(t, v(t))=w \frac{\partial p(t, \theta u+(1-\theta) v)}{\partial u}
$$

Testing this equation with $w_{t}$ yields

$$
\frac{1}{2} \frac{d}{d t}\left(\left\|w_{t}\right\|^{2}+\|\nabla w\|^{2}\right)+\left\|w_{t}\right\|^{2}=\left(w \frac{\partial p(t, \theta u+(1-\theta) v)}{\partial u}, w_{t}\right)
$$

Assumption (H5) gives inequality

$$
\frac{1}{2} \frac{d}{d t}\left(\left\|w_{t}\right\|^{2}+\|\nabla w\|^{2}\right) \leq C \int_{\Omega} w\left(1+|u|^{4}+|v|^{4}\right) w_{t} d x
$$

Then by using the Hölder inequality with exponents $\frac{1}{6}, \frac{1}{3}, \frac{1}{2}$ and the Sobolev embed$\operatorname{ding} H_{0}^{1} \hookrightarrow L^{6}$ we obtain

$$
\frac{d}{d t}\left(\left\|w_{t}\right\|^{2}+\|\nabla w\|^{2}\right) \leq C\left(\|\nabla w\|^{2}+\left\|w_{t}\right\|^{2}\right)\left(1+\|u\|_{L^{12}}^{4}+\|v\|_{L^{12}}^{4}\right)
$$

Because $v, u$ are Shatah-Struwe solution, i.e. $u, v \in L_{l o c}^{4}\left([0, \infty) ; L^{12}\right)$, it is possible to use integral form of the Gröwall inequality which gives us

$$
\|\nabla w\|^{2}+\left\|w_{t}\right\|^{2} \leq\left(\left\|\nabla w_{0}\right\|^{2}+\left\|w_{1}\right\|^{2}\right) \exp \left(C\left(t+\int_{0}^{t}\|v\|_{L_{12}}^{4}+\|w\|_{L_{12}}^{4} d t\right)\right)
$$

for $t \in[0, \infty)$, hence the assertion follows.

Lemma 5.9 Every weak solution of problem (3.1) is of Galerkin type if and only it is a Shatah-Struwe solution. Moreover for every $t>0$ there exists a constant $C_{t}>0$ such that for every solution $u$ of (3.1), with arbitrary $p \in \mathcal{H}_{[0,1]}$ in the place of $f_{\varepsilon}$, contained in the absorbing set $B_{0}$, it holds that

$$
\|u\|_{L^{4}\left(0, t ; L^{12}\right)} \leq C_{t} .
$$

Proof Let $u$ be the solution of the Galerkin type with the initial data $\left(u_{0}, u_{1}\right) \in \mathcal{E}_{0}$. From assumption (H5) we see that

$$
\|p(t, u)\| \leq C\left(1+\left\||u|^{5-\kappa}\right\|\right)=C\left(1+\|u\|_{L^{2(5-\kappa)}}^{5-\kappa}\right) \leq C\left(1+\|u\|_{L^{10}}^{5-\kappa}\right) .
$$


We assume that $t \in[0,1]$. From the Hölder inequality we obtain

$$
\begin{aligned}
& \int_{0}^{t}\|p(s, u)\| d s \leq C t+C \int_{0}^{t}\|u\|_{L^{10}}^{5-\kappa} d s \\
& \quad \leq C\left(\left(\int_{0}^{t}\|u\|_{L^{10}}^{5} d s\right)^{\frac{5-\kappa}{5}}\left(\int_{0}^{t} 1 d t\right)^{\frac{\kappa}{5}}+t\right) \\
& \quad=C\left(\|u\|_{L^{5}\left(0, t ; L^{10}\right)}^{5-\kappa} t^{\frac{\kappa}{5}}+t\right) \leq C\left(\|u\|_{L^{5}\left(0, t ; L^{10}\right)}^{5-\kappa}+1\right) t^{\frac{\kappa}{5}} \\
& \quad \leq C R^{\frac{1}{5}}\left(\|u\|_{L^{4}\left(0, t ; L^{12}\right)}^{4-\frac{4 \kappa}{5}}+1\right) t^{\frac{\kappa}{5}}
\end{aligned}
$$

where $R$ is the bound of the $L^{\infty}\left(0, t ; H_{0}^{1}\right)$ norm of $u$. We split $u$ as the sum $u=v+w$ where $v, w$ solve the following problems

$$
\left\{\begin{array} { l } 
{ v _ { t t } + v _ { t } - \Delta v = 0 , } \\
{ v ( t , x ) = 0 \text { for } x \in \partial \Omega , } \\
{ v ( 0 , x ) = u _ { 0 } ( x ) , } \\
{ v _ { t } ( 0 , x ) = u _ { 1 } ( x ) , }
\end{array} \quad \left\{\begin{array}{l}
w_{t t}+w_{t}-\Delta w=p_{\epsilon}(t, u), \\
w(t, x)=0 \text { for } x \in \partial \Omega, \\
w(0, x)=0, \\
w_{t}(0, x)=0 .
\end{array}\right.\right.
$$

From the Strichartz estimate in Lemma 5.2 we deduce

$$
\|v\|_{L^{4}\left(0, t ; L^{12}\right)} \leq C_{1}\left\|\left(u_{0}, u_{1}\right)\right\|_{\mathcal{E}_{0}},
$$

and

$$
\|w\|_{L^{4}\left(0, t ; L^{12}\right)} \leq C R^{\frac{1}{5}}\left(\|w\|_{L^{4}\left(0, t ; L^{12}\right)}^{4-\frac{4 \kappa}{5}}+\left(C_{1}\left\|\left(u_{0}, u_{1}\right)\right\| \mathcal{E}_{0}\right)^{4-\frac{4 \kappa}{5}}+1\right) t^{\frac{\kappa}{5}} .
$$

We define the function $Y(t)=\|w\|_{L^{4}\left(0, t ; L^{12}\right)}$ for $t \in[0,1]$. Formally we do not know if this function is well defined, so to make the proof rigorous we should proceed for Galerkin approximation, cf. [27]. We continue the proof in formal way. The function $Y(t)=\|w\|_{L^{4}\left(0, t ; L^{12}\right)}$ is continuous with $Y(0)=0$ and

$$
Y(t) \leq C R^{\frac{1}{5}}\left(Y(t)^{4-\frac{4 \kappa}{5}}+\left(C_{1}\left\|\left(u_{0}, u_{1}\right)\right\|_{\mathcal{E}_{0}}\right)^{4-\frac{4 \kappa}{5}}+1\right) t^{\frac{\kappa}{5}}
$$

We define

$$
t_{\max }^{\frac{\kappa}{5}}=\min \left\{\frac{1}{2 C R^{\frac{1}{5}}\left(\left(C_{1} S\right)^{4-\frac{4 \kappa}{5}}+2\right)}, 1\right\}, \text { where } S \geq\left\|\left(u_{0}, u_{1}\right)\right\|_{\mathcal{E}_{0}}
$$

Now we will use continuation method to prove that the estimate $Y(t) \leq 1$ holds on the interval $\left[0, t_{\max }\right]$. The argument follows the scheme of the proof from [40, Proposition 1.21]. Defining the logical predicates $H(t)=(Y(t) \leq 1)$ and $C(t)=\left(Y(t) \leq \frac{1}{2}\right)$ we observe that following facts hold 
- $C(0)$ is true.

- If $C\left(s_{0}\right)$ for some $s_{0}$ is true then $H(s)$ is true in some neighbourhood of $s_{0}$.

- If $s_{n} \rightarrow s_{0}$ and $C\left(s_{n}\right)$ holds for every $n$ then $C\left(s_{0}\right)$ is true.

- $H(t)$ implies $C(t)$ for $t \in\left[0, t_{\max }\right]$, indeed

$$
\begin{aligned}
Y(t) & \leq C R^{\frac{1}{5}}\left(\left(C_{1}\left\|u_{0}, u_{1}\right\|_{\mathcal{E}_{0}}\right)^{4-\frac{4 \kappa}{5}}+1+Y(t)^{4-\frac{4 \kappa}{5}}\right) t^{\frac{\kappa}{5}} \\
& \leq C R^{\frac{1}{5}}\left(C_{1}\left\|u_{0}, u_{1}\right\|_{\mathcal{E}_{0}}+2\right)^{\frac{4-\kappa}{5}} t_{\max }^{\frac{\kappa}{5}} \leq \frac{1}{2} .
\end{aligned}
$$

The continuation argument implies that $C(t)$ holds for $t \in\left[0, t_{\max }\right]$. From the triangle inequality we conclude that

$$
\|u\|_{L^{4}\left(0, t_{\max } ; L^{12}\right)} \leq C_{1}\left\|\left(u_{0}, u_{1}\right)\right\|_{\mathcal{E}_{0}}+1
$$

Observe that $t_{\max }$ and $C_{1}$ are independent of choice of $p \in \mathcal{H}_{[0,1]}$. Because all trajectories are bounded in the $\mathcal{E}_{0}$ norm, cf. Proposition 4.5, we deduce that $\|u\|_{L^{4}\left(0, t, L^{12}\right)}$ is well defined for every $t>0$. Moreover if $\left(u(t), u_{t}(t)\right) \in B_{0}$ for every $t \geq 0$, then the bound on the $\mathcal{E}_{0}$ norm of the solution is uniform and we deduce the bound $\|u\|_{L^{4}\left(0, t, L^{12}\right)} \leq C_{t}$ with $C_{t}$ independent of $p$.

Remark 5.10 As a consequence of Proposition 5.8 and Lemma 5.9 for every $\left(u_{0}, u_{1}\right) \in$ $\mathcal{E}_{0}$, the weak solution of Galerkin type of problem (3.1) is unique.

Lemma 5.11 If the weak solution $\left(u, u_{t}\right)$ of problem (3.1) is of Galerkin type, then for every $T>0$ it belongs to the space $C\left([0, T] ; \mathcal{E}_{0}\right)$.

Proof The proof follows the arguments of [27, Proposition 3.3]. They key fact is that Galerkin (or equivalently, Shatah-Struwe) solutions satisfy the energy equation. Let $t_{n} \rightarrow t$ and let $T>\sup _{n \in N}\left\{t_{n}\right\}$. Clearly, $\left(u, u_{t}\right) \in C_{w}\left([0, T] ; \mathcal{E}_{0}\right)$ and hence $\left(u\left(t_{n}\right), u_{t}\left(t_{n}\right)\right) \rightarrow\left(u(t), u_{t}(t)\right)$ weakly in $\mathcal{E}_{0}$. To deduce that this convergences is strong we need to show that $\left\|\left(u\left(t_{n}\right), u_{t}\left(t_{n}\right)\right)\right\|_{\mathcal{E}_{0}} \rightarrow\left\|\left(u(t), u_{t}(t)\right)\right\|_{\mathcal{E}_{0}}$. To this end we will use the energy equation

$$
\left\|\left(u(t), u_{t}(t)\right)\right\|_{\mathcal{E}_{0}}^{2}-\left\|\left(u\left(t_{n}\right), u_{t}\left(t_{n}\right)\right)\right\|_{\mathcal{E}_{0}}^{2}=2 \int_{t_{n}}^{t}\left(p(s, u(s)), u_{t}\right)-\left\|u_{t}(s)\right\|^{2} d s .
$$

Then

$$
\left|\left\|\left(u(t), u_{t}(t)\right)\right\|_{\mathcal{E}_{0}}^{2}-\left\|\left(u\left(t_{n}\right), u_{t}\left(t_{n}\right)\right)\right\|_{\mathcal{E}_{0}}^{2}\right| \leq C R\left((R+1)\left|t-t_{n}\right|+\|u\|_{L^{5}\left(t_{n}, t ; L^{10}\right)}\right)
$$

where $R$ is the bound of the $L^{\infty}\left(0, T ; L^{2}\right)$ norm of $u_{t}$. The right side tends to zero as $t_{n} \rightarrow t$ which proves the assertion.

\subsection{Non-autonomous dynamical system.}

We will denote by 
$\left(u(t), u_{t}(t)\right)=\varphi_{\varepsilon}(t, p)\left(u_{0}, u_{1}\right)$ the map which gives the Galerkin-type solution of (3.1) with $p \in \mathcal{H}\left(f_{\varepsilon}\right)$ as the right-hand side and the initial conditions $u(0)=u_{0}$, $u(0)=u_{1}$. We remind the definitions of non-autonomous dynamical system and cocycle.

Definition 5.12 Let $X, \Sigma$ be metric spaces. Assume that $\left\{\theta_{t}\right\}_{t \geq 0}$ is a semigroup in $\Sigma$ and $\varphi: \mathbb{R}^{+} \times \Sigma \rightarrow C(X)$ is a continuous map. Let the following conditions hold

- $\varphi(0, \sigma)=\operatorname{Id}_{X}$ for every $\sigma \in \Sigma$.

- The map $\mathbb{R}^{+} \times \Sigma \ni(t, \sigma) \rightarrow \varphi(t, \sigma) x \in X$ is continuous for every $x$.

- For every $t, s \geq 0$ and $\sigma \in \Sigma$ the cocycle property holds

$$
\varphi(t+s, \sigma)=\varphi\left(t, \theta_{s} \sigma\right) \varphi(s, \sigma)
$$

Then the pair $(\varphi, \theta)$ is called a non-autonomous dynamical (NDS) and map $\varphi$ a cocycle semiflow.

The next result shows that $\varphi_{\varepsilon}$ is an NDS with $X=\mathcal{E}$ and $\Sigma=\mathcal{H}\left(f_{\varepsilon}\right)$.

Proposition 5.13 The mapping $\varphi_{\varepsilon}: \mathbb{R} \times \mathcal{H}\left(f_{\varepsilon}\right) \rightarrow C(\mathcal{E})$ together with time translation $\theta_{t} p_{\varepsilon}=p_{\varepsilon}(\cdot+t)$ constitute a non-autonomous dynamical system.

Proof Property $\varphi(0, p)=\operatorname{Id}_{\mathcal{E}_{0}}$ and the cocycle property are obvious from definition of $\varphi_{\varepsilon}$ and $\theta_{t}$. Let $\left(u_{0}^{n}, u_{1}^{n}\right) \rightarrow\left(u_{0}, u_{1}\right)$ in $\mathcal{E}_{0}, p_{\varepsilon}^{n} \rightarrow p_{\varepsilon}$ in the metric of $d_{C\left(\mathbb{R} ; C^{1}(\mathbb{R})\right)}$ restricted to $\mathcal{H}\left(f_{\varepsilon}\right), t_{n} \rightarrow t$ and let $\left\{u^{n}\right\}_{n=1}^{\infty}$ and $u$ be the Galerkin type weak solutions of the problems governed by the equations

$$
\begin{aligned}
& u_{t t}^{n}+u_{t}^{n}-\Delta u^{n}=p_{\varepsilon}^{n}\left(t, u^{n}\right), \\
& u_{t t}+u_{t}-\Delta u=p_{\varepsilon}(t, u),
\end{aligned}
$$

with the boundary data $u^{n}=u=0$ on $\partial \Omega$ and initial data $\left(u^{n}(0), u_{t}^{n}(0)\right)=\left(u_{0}^{n}, u_{1}^{n}\right) \in$ $\mathcal{E}_{0}$ and $\left(u(0), u_{t}(0)\right)=\left(u_{0}, u_{1}\right) \in \mathcal{E}_{0}$. Choose $T>0$ such that $T>\sup _{n \in \mathbb{N}}\left\{t_{n}\right\}$. The following bounds hold

$$
\begin{gathered}
\left\|\nabla u^{n}(t)\right\|_{L^{2}} \leq C, \quad\|\nabla u(t)\|_{L^{2}} \leq C, \\
\left\|u_{t}^{n}(t)\right\|_{L^{2}} \leq C, \quad\left\|u_{t}(t)\right\|_{L^{2}} \leq C, \\
\left\|u_{t t}^{n}(t)\right\|_{H^{-1}} \leq C, \quad\left\|u_{t t}(t)\right\|_{H^{-1}} \leq C .
\end{gathered}
$$

for $t \in[0, T]$ with a constant $C>0$. Moreover, the following bounds also hold

$$
\left\|u^{n}\right\|_{L^{4}\left(0, T ; L^{12}\right)} \leq C,\|u\|_{L^{4}\left(0, T ; L^{12}\right)} \leq C .
$$

This means that, for a subsequence

$$
\begin{gathered}
u^{n} \rightarrow v \text { weakly-* in } L^{\infty}\left(0, T ; H_{0}^{1}\right), \\
u_{t}^{n} \rightarrow v_{t} \text { weakly-* in } L^{\infty}\left(0, T ; L^{2}\right), \\
u_{t t}^{n} \rightarrow v_{t t} \text { weakly-* in } L^{\infty}\left(0, T ; H^{-1}\right),
\end{gathered}
$$


for a certain function $v \in L^{\infty}\left(0, T ; H_{0}^{1}\right)$ with $v_{t} \in L^{\infty}\left(0, T ; L^{2}\right)$ and $v_{t t} \in$ $L^{\infty}\left(0, T ; H^{-1}\right)$. By Lemma $5.11 u^{n}, u \in C\left([0, T] ; \mathcal{E}_{0}\right)$. Moreover $v \in C\left([0, T] ; L^{2}\right) \cap$ $C_{w}\left([0, T] ; H_{0}^{1}\right)$ and $v_{t} \in C\left([0, T] ; H^{-1}\right) \cap C_{w}\left([0, T] ; L^{2}\right)$, cf. [41, Lemma 1.4, page 263]. We will show that $v=u$ for $t \in[0, T]$. Note that for every $w \in L^{2}$

$$
\left(u^{n}(0), w\right)=\left(u^{n}(t), w\right)-\int_{0}^{t}\left(u_{t}^{n}(s), w\right) d s .
$$

Integrating with respect to $t$ between 0 and $T$ and exchanging the order of integration we obtain

$$
T\left(u_{0}^{n}, w\right)=\int_{0}^{T}\left(u^{n}(t), w\right) d t-\int_{0}^{T}\left(u_{t}^{n}(s),(T-s) w\right) d s .
$$

Passing to the limit we obtain

$$
T\left(u_{0}, w\right)=\int_{0}^{T}(v(t), w) d t-\int_{0}^{T}\left(v_{t}(s),(T-s) w\right) d s=T(v(0), w),
$$

whence $v(0)=u_{0}$. It is straightforward to see that $u^{n}(t) \rightarrow v(t)$ weakly in $H_{0}^{1}$ for every $t \in[0, T]$. Similar reasoning for $u_{t}^{n}$ allows us to deduce that $v_{t}(0)=u_{1}$ and $u_{t}^{n}(t) \rightarrow v_{t}(t)$ weakly in $L^{2}$ for every $t \in[0, T]$. Now we have to show that $v$ satsfies (5.6). Indeed, weak form of (5.5) is as follows

$$
\begin{aligned}
& \int_{0}^{T}\left\langle u_{t t}^{n}(t), w(t)\right\rangle_{H^{-1} \times H_{0}^{1}} d t+\int_{0}^{T}\left(u_{t}^{n}(t), w(t)\right) d t+\int_{0}^{T}\left(\nabla u^{n}(t), \nabla w(t)\right) d t \\
& \quad=\int_{0}^{T} \int_{\Omega} p_{\varepsilon}^{n}\left(t, u^{n}(x, t)\right) w(t) d x d t
\end{aligned}
$$

for every $w \in L^{2}\left(0, T ; H_{0}^{1}\right)$. It suffices only to pass to the limit on the right-hand side. Fix $t \in[0, T]$ and $w \in H_{0}^{1}$. By the compact embedding $H_{0}^{1} \hookrightarrow L^{p}$ for $p \in[1,6)$ it holds that $u^{n}(\cdot, t) \rightarrow u(\cdot, t)$ strongly in $L^{6-\frac{6}{5} \kappa}$ and, for a subsequence, $u^{n}(x, t) \rightarrow$ $u(x, t)$ for a.e. $x \in \Omega$ and $\left|u^{n}(x, t)\right| \leq g(x)$ with $g \in L^{6-\frac{6}{5} \kappa}$, where $g$ can also depend on $t$. Hence

$$
p_{\varepsilon}^{n}\left(t, u^{n}(x, t)\right) w(x) \rightarrow p_{\varepsilon}(t, u(x, t)) w(x) \text { for a.e. } x \in \Omega,
$$

moreover, by the Young inequality,

$$
\begin{aligned}
\left|p_{\varepsilon}^{n}\left(t, u^{n}(x, t)\right) w(x)\right| & \leq C\left(1+\left|u^{n}(x, t)\right|^{5-\kappa}\right)|w(x)| \\
& \leq|w(x)|^{6}+C\left(1+g(x)^{6-\frac{6}{5} \kappa}\right) \in L^{1} .
\end{aligned}
$$

We can use the Lebesgue dominated convergence theorem to obtain

$$
\lim _{n \rightarrow \infty} \int_{\Omega} p_{\varepsilon}^{n}\left(t, u^{n}(x, t)\right) w(x) d x=\int_{\Omega} p_{\varepsilon}(t, u(x, t)) w(x) d x \quad \text { for a.e. } \quad t \in(0, T) .
$$


Now let $w \in L^{2}\left(0, T ; H_{0}^{1}\right)$. The following holds

$$
\begin{aligned}
& \left|\int_{\Omega} p_{\varepsilon}^{n}\left(t, u^{n}(x, t)\right) w(x, t) d x\right| \\
& \quad \leq C \int_{\Omega}\left(1+\left|u^{n}(x, t)\right|^{5}\right)|w(x, t)| d x \leq C\|w(t)\|_{L^{6}}\left(1+\left\|u^{n}(t)\right\|_{L^{6}}^{5}\right) \\
& \quad \leq C\|w(t)\|_{H_{0}^{1}}\left(1+\left\|u^{n}(t)\right\|_{H_{0}^{1}}^{5}\right) \leq C\|w(t)\|_{H_{0}^{1}} \in L^{1}(0, T),
\end{aligned}
$$

whence we can pass to the limit in the nonlinear term. The fact that the $L^{4}\left(0, T ; L^{12}\right)$ estimate on $u^{n}$ is independent of $n$ implies that $v$ satisfies the same estimate which ends the proof that $u=v$.

We must show that $\left\|\left(u^{n}\left(t_{n}\right), u_{t}^{n}\left(t_{n}\right)\right)-\left(u(t), u_{t}(t)\right)\right\|_{\mathcal{E}_{0}} \rightarrow 0$ We already know that $u^{n}(t) \rightarrow u(t)$ weakly in $H_{0}^{1}$ and $u_{t}^{n}(t) \rightarrow u_{t}(t)$ weakly in $L^{2}$ for every $t \in[0, T]$. We will first prove that these convergences are strong. To this end let $w^{n}=u^{n}-u$. The following holds

$$
w_{t t}^{n}+w_{t}^{n}-\Delta w^{n}=p_{\varepsilon}^{n}\left(t, u^{n}\right)-p_{\varepsilon}(t, u)
$$

Testing this equation with $w_{t}^{n}$ we obtain

$$
\frac{1}{2} \frac{d}{d t}\left\|\left(w^{n}(t), w_{t}^{n}(t)\right)\right\|_{\mathcal{E}_{0}}^{2}+\left\|w_{t}^{n}(t)\right\|^{2}=\int_{\Omega}\left(p_{\varepsilon}^{n}\left(t, u^{n}\right)-p_{\varepsilon}(t, u)\right) w_{t}^{n}(t) d x .
$$

Simple computations lead us to

$$
\begin{aligned}
& \frac{d}{d t}\left\|\left(w^{n}(t), w_{t}^{n}(t)\right)\right\|_{\mathcal{E}_{0}}^{2} \\
& \leq \frac{1}{2} \int_{\Omega}\left(p_{\varepsilon}^{n}(t, u)-p_{\varepsilon}(t, u)\right)^{2} d x \\
& \quad+2 \int_{\Omega}\left(p_{\varepsilon}^{n}\left(t, u^{n}\right)-p_{\varepsilon}^{n}(t, u)\right) w_{t}^{n}(t) d x .
\end{aligned}
$$

After integration from 0 to $t$ we obtain

$$
\begin{aligned}
& \left\|\left(w^{n}(t), w_{t}^{n}(t)\right)\right\|_{\mathcal{E}_{0}}^{2} \leq\left\|\left(u_{0}^{n}-u^{n}, u_{1}^{n}-u_{1}\right)\right\|_{\mathcal{E}_{0}}^{2} \\
& \quad+\frac{1}{2} \int_{0}^{T} \int_{\Omega}\left(p_{\varepsilon}^{n}(s, u)-p_{\varepsilon}(s, u)\right)^{2} d x d s \\
& \quad+2 \int_{0}^{T} \int_{\Omega}\left|\left(p_{\varepsilon}^{n}\left(s, u^{n}\right)-p_{\varepsilon}^{n}(s, u)\right) w_{t}^{n}(s)\right| d x d s .
\end{aligned}
$$

We must show that the right hand side in the above inequality tends to zero as $n$ goes to infinity. Clearly, the first term tends to zero. To deal with the second term note that $p_{\varepsilon}^{n}(s, u) \rightarrow p_{\varepsilon}(s, u)$ for almost every $(x, s) \in \Omega \times(0, T)$. Moreover by (H3)

$$
\left(p_{\varepsilon}^{n}(s, u)-p_{\varepsilon}(s, u)\right)^{2} \leq C,
$$


and the Lebesgue dominated convergence theorem implies the assertion. We deal with the last term. By the mean value theorem and (H5) we obtain

$$
\left|\left(p_{\varepsilon}^{n}\left(s, u^{n}\right)-p_{\varepsilon}^{n}(s, u)\right) w_{t}^{n}(s)\right| \leq C\left|u^{n}(s)-u(s)\right|\left(1+\left|u^{n}(s)\right|^{4-\kappa}+|u(s)|^{4-\kappa}\right)\left|w_{t}^{n}(s)\right|
$$

From the compact embedding $H_{0}^{1} \hookrightarrow L^{q}$ for $q \in[1,6)$ by the Aubin-Lions lemma, cf. [39, Corollary 4] we know that, for a subsequence, $u^{n}-u \rightarrow 0$ in $C\left([0, T] ; L^{q}\right)$ with $q \in[1,6)$. This motivates the use of the Hölder inequality with exponents $q=\frac{12}{2+\kappa}<6, p=\frac{12}{4-\kappa}, r=2$, which yields

$$
\begin{aligned}
& \int_{\Omega}\left|\left(p_{\varepsilon}^{n}\left(s, u^{n}\right)-p_{\varepsilon}^{n}(s, u)\right) w_{t}^{n}(s)\right| d x \\
& \quad \leq C\left\|u^{n}(s)-u(s)\right\|_{L^{2}}\left\|w_{t}^{n}(s)\right\|_{L^{2}} \\
& \quad+C\left\|u^{n}(s)-u(s)\right\|_{L^{\frac{12}{2+\kappa}}}\left(\left\|u^{n}(s)\right\|_{L^{12}}^{4-\kappa}+\|u(s)\|_{L^{12}}^{4-\kappa}\right)\left\|w_{t}^{n}(s)\right\|_{L^{2}} .
\end{aligned}
$$

Using the fact that $\left\|w_{t}^{n}(s)\right\|_{L^{2}} \leq\left\|u_{t}^{n}(s)\right\|_{L^{2}}+\left\|u_{t}(s)\right\|_{L^{2}} \leq C$, after integration in time we obtain

$$
\begin{aligned}
& \int_{0}^{T} \int_{\Omega}\left|\left(p_{\varepsilon}^{n}\left(s, u^{n}\right)-p_{\varepsilon}^{n}(s, u)\right) w_{t}^{n}(s)\right| d x d s \leq C T \sup _{s \in[0, T]}\left\|u^{n}(s)-u(s)\right\|_{L^{2}} \\
& \quad+C \sup _{s \in[0, T]}\left\|u^{n}(s)-u(s)\right\|_{L^{2+\kappa}}\left(\int_{0}^{T}\left\|u^{n}(s)\right\|_{L^{12}}^{4-\kappa} d s+\int_{0}^{T}\|u(s)\|_{L^{12}}^{4-\kappa} d s\right) .
\end{aligned}
$$

The last two time integrals are bounded from (5.7) by a constant independent of $n$, whence the whole expression converges to zero.

Now, the triangle inequality implies

$$
\begin{aligned}
& \left\|\nabla u^{n}\left(t_{n}\right)-\nabla u(t)\right\|_{L^{2}}^{2}+\left\|u_{t}^{n}\left(t_{n}\right)-u_{t}(t)\right\|_{L^{2}}^{2} \\
& \leq 2\left(\left\|\nabla u^{n}\left(t_{n}\right)-\nabla u\left(t_{n}\right)\right\|_{L^{2}}^{2}+\left\|u_{t}^{n}\left(t_{n}\right)-u_{t}\left(t_{n}\right)\right\|_{L^{2}}^{2}\right) \\
& \quad+2\left(\left\|\nabla u\left(t_{n}\right)-\nabla u(t)\right\|_{L^{2}}^{2}+\left\|u_{t}\left(t_{n}\right)-u_{t}(t)\right\|_{L^{2}}^{2}\right),
\end{aligned}
$$

where both terms tend to zero, the first one by passing to the limit in (5.8) and the second one by Lemma 5.11 which completes the proof.

\section{Existence and regularity of non-autonomous attractors.}

\subsection{Abstract results on existence and structure of non-autonomous attractors.}

In this subsection we remind the definitions of uniform and cocycle attractors, and the results on their existence and relations between them. These results can be found for example in $[7,28]$. We remind that the Hausdorff semidistance in the metric space $(X, d)$ between the two sets $A, B \subset X$ is defined as 


$$
\operatorname{dist}_{X}(A, B)=\sup _{x \in A} \inf _{y \in B} d(x, y) .
$$

Definition 6.1 The set $\mathcal{A} \subset X$ is called the uniform attractor for the cocycle $\varphi$ on $X$ if $\mathcal{A}$ is smallest compact set such that for every bounded sets $B \subset X$ and $\Upsilon \subset \Sigma$ it holds that

$$
\lim _{t \rightarrow \infty} \sup _{\sigma \in \Upsilon} \operatorname{dist}_{X}(\varphi(t, \sigma) B, \mathcal{A}) \rightarrow 0
$$

Definition 6.2 Let $(\varphi, \theta)$ be an NDS such that $\theta$ is a group i.e $\Sigma$ is invariant for every $\theta_{t}$. Then we call the family of compact sets $\{\mathcal{A}(\sigma)\}_{\sigma \in \Sigma}$ the cocycle atractor if

- $\{\mathcal{A}(\sigma)\}_{\sigma \in \Sigma}$ is invariant under the $\operatorname{NDS}(\varphi, \theta)$, i.e.,

$$
\varphi(t, \sigma) \mathcal{A}(\sigma)=\mathcal{A}\left(\theta_{t} \sigma\right) \text { for every } t \geq 0
$$

- $\{\mathcal{A}(\sigma)\}_{\sigma \in \Sigma}$ pullback attracts all bounded subsets $B \subset X$, i.e.,

$$
\left.\lim _{t \rightarrow \infty} \operatorname{dist}_{X}\left(\varphi\left(t, \theta_{-t} \sigma\right) B, \mathcal{A}(\sigma)\right)\right)=0
$$

Remark 6.3 If for some $\sigma \in \Sigma$ we consider the mapping $S(t, \tau)=\varphi\left(t-\tau, \theta_{\tau} \sigma\right)$ for an NDS $(\varphi, \theta)$ then the family of mappings $\{S(t, \tau): t \geq \tau\}$ forms an evolution process. Let $\{\mathcal{A}(\sigma)\}_{\sigma \in \Sigma}$ be a cocycyle atrator for NDS. Then $\mathcal{A}(t)=\mathcal{A}\left(\theta_{t} \sigma\right)$ is called a pullback atractor for $S(t, \tau)$.

Definition 6.4 We say that the $\operatorname{NDS}(\varphi, \theta)$ is uniformly asymptotically compact if there exist a compact set $K \subset X$ such that

$$
\lim _{t \rightarrow \infty} \sup _{\sigma \in \Upsilon} \operatorname{dist}_{X}(\varphi(t, \sigma) B, K)=0 .
$$

Theorem 6.5 [7, Theorem 3.12.] Let $\operatorname{NDS}(\varphi, \theta)$ be such that $\theta$ is a group. Assume that $(\varphi, \theta)$ is uniformly asymptotically compact, and $\Sigma$ is compact. Then the uniform and cocycle attractors exist and it holds that

$$
\bigcup_{\sigma \in \Sigma} \mathcal{A}(\sigma)=\mathcal{A}
$$

where $\{\mathcal{A}(\sigma)\}_{\sigma \in \Sigma}$ is the cocycle atractor and $\mathcal{A}$ is the uniform atractor.

Definition 6.6 Let $(\varphi, \theta)$ be an NDS such that $\theta$ is a group. We call $\xi: \mathbb{R} \rightarrow X$ a global solution through $x$ and $\sigma$ if, for all $t \geq s$ it satisfies

$$
\varphi\left(t-s, \theta_{s} \sigma\right) \xi(s)=\xi(t) \text { and } \xi(0)=x .
$$

Moreover we say that a subset $\mathcal{M} \subset X$ is lifted-invariant if for each $x \in \mathcal{M}$ there exist $\sigma$ and bounded global solution $\xi: \mathbb{R} \rightarrow X$ through $x$ and $\sigma$. 
Theorem 6.7 [7, Proposition 3.21] Let $\operatorname{NDS}(\varphi, \theta)$ be such that $\theta$ is a group. Assume that $(\varphi, \theta)$ is uniformly asymptotically compact, and $\Sigma$ is compact. Then the uniform attractor $\mathcal{A}$ is the maximal bounded lifted-invariant set of the $\operatorname{NDS}(\varphi, \theta)$.

\subsection{Uniform and cocycle attractors for the NDS $\varphi_{\epsilon}$.}

We show the existence and regularity of uniform and cocycle attractors, and relation between them for the NDS given by the Galerkin (or equivalently Shatah-Struwe) solutions of our problem with subquintic nonlinearity. The key property is the uniform asymptotic compactness. To obtain it, we start from the result which states that the solution can be split into the sum of two functions: one that decays to zero, and another one which is more smooth than the initial data.

Lemma 6.8 Let $u$ be the Shatah-Struwe solution of (3.1) such that $u(t) \in B_{0}$ for every $t \geq 0$, where $B_{0}$ is the absorbing set from Proposition 4.5. There exists a finite and increasing sequence $\beta_{0}, \ldots, \beta_{k}$ with $\beta_{0}=0, \beta_{k}=1$ and the constants $C, C_{R}, \alpha>0$ such that if $i \in\{0, \ldots, k-1\}$ and $\left\|\left(u(t), u_{t}(t)\right)\right\|_{\mathcal{E}_{\beta_{i}}} \leq R$ for every $t \in[0, \infty)$, then $u$ can be represented as the sum of two functions $v, w$ satisfying

$$
\begin{gathered}
u(t)=v(t)+w(t),\left\|\left(v(t), v_{t}(t)\right)\right\|_{\mathcal{E}_{\beta_{i}}} \leq\left\|\left(u_{0}, u_{1}\right)\right\|_{\mathcal{E}_{\beta_{i}}} C e^{-\alpha t} \\
\text { and }\left\|\left(w(t), w_{t}(t)\right)\right\|_{\mathcal{E}_{\beta_{i+1}} \leq C_{R} \text { for } i \in\{0, \ldots, k-1\} .}
\end{gathered}
$$

Moreover the constants $C, C_{R}, \alpha$ are independent of the choice of $p_{\varepsilon} \in \mathcal{H}_{[0,1]}$ treated as the right-hand side in equation (3.1).

Proof Our first aim is to obtain the relation between $\beta_{i}$ and $\beta_{i+1}$ such that if for every $t \in[0, \infty)$ the bound $\left\|\left(u(t), u_{t}(t)\right)\right\|_{\mathcal{E}_{\beta_{i}}} \leq R$ holds, then $p_{\varepsilon}(t, u) \in$ $L_{l o c}^{1}\left([0, \infty) ; H^{\beta_{i+1}}\right)$. To this end we first interpolate between $L^{q}$ and $W^{1, s}$, whence, from the Gagliardo-Nirenberg inequality we obtain

$$
\left\|p_{\varepsilon}(t, u)\right\|_{H^{\alpha}} \leq C\left\|\nabla p_{\varepsilon}(t, u)\right\|_{L^{s}}^{\theta}\left\|p_{\varepsilon}(t, u)\right\|_{L^{q}}^{1-\theta}+C\left\|p_{\varepsilon}(t, u)\right\|_{L^{q}}
$$

with $\alpha \leq \theta \leq 1, \frac{1}{2}=\frac{\alpha}{3}+\left(\frac{1}{s}-\frac{1}{3}\right) \theta+\frac{1-\theta}{q}$ and $s<2$. From the chain rule and the Hölder inequality with conjugate exponents $p$ and $p^{\prime}$ we deduce

$$
\begin{aligned}
& \left\|p_{\varepsilon}(t, u)\right\|_{H^{\alpha}} \\
& \leq C\left(\int_{\Omega}\left|\frac{\partial p_{\varepsilon}}{\partial u}(t, u)\right|^{s p^{\prime}} d x\right)^{\frac{\theta}{s p^{\prime}}}\left(\int_{\Omega}|\nabla u|^{s p} d x\right)^{\frac{\theta}{s p}}\left\|p_{\varepsilon}(t, u)\right\|_{L^{q}}^{1-\theta} \\
& \quad+C\left\|p_{\varepsilon}(t, u)\right\|_{L^{q}} .
\end{aligned}
$$


From assumption (H5), the Cauchy inequality, and the fact that solution $u$ is included in the absorbing set, taking $s p=2, s p^{\prime}=3, \theta=\frac{1}{2}$ we get the inequality

$$
\begin{gathered}
\left\|p_{\varepsilon}(t, u)\right\|_{H^{\alpha}} \leq C(R)\left(\left(\int_{\Omega}|u|^{12} d x\right)^{\frac{1}{3}}+\left(\int_{\Omega}|u|^{(5-\kappa) q} d x\right)^{\frac{1}{q}}+1\right) \\
\text { with } \alpha=\frac{3}{2}\left(\frac{1}{2}-\frac{1}{q}\right), \alpha<\frac{1}{2} .
\end{gathered}
$$

The choice of $s, p, \theta$ is motivated by the fact that we need the terms which we can control to appear on the right hand side of (6.1) after time integration. In the first step of the bootstrap argument this can be either the Strichartz norm $L^{4}\left(L^{12}\right)$ of the solution $u$, or its energy norm $L^{\infty}\left(H_{0}^{1}\right)$. In the consecutive steps of the bootstrap argument we need the terms on the right-hand side which can be controlled having the bounds on $L^{4}\left(W^{\beta_{i}, 12}\right)$ and $L^{\infty}\left(H_{0}^{\beta_{i}+1}\right)$ norms of the solution. Now we will inductively describe the sequence $\beta_{1} \ldots, \beta_{k-1}$ starting with $\beta_{1}$. If we set $\frac{5-\kappa}{10} \leq \frac{1}{q}<\frac{1}{2}$ in inequality (6.1), we obtain

$$
\begin{aligned}
& \int_{t_{0}}^{t_{0}+h}\left\|p_{\varepsilon}(t, u)\right\|_{H^{\beta_{1}}} d t \\
& \quad \leq C(R)\left(\|u\|_{L^{4}\left(t_{0}, t_{0}+h ; L^{12}\right)}^{4}+\|u\|_{L^{5}\left(t_{0}, t_{0}+h ; L^{10}\right)}^{5}+h\right) \\
& \quad \leq C(h, R) .
\end{aligned}
$$

We observe that $\beta_{1} \in(0, \delta)$, for some $\delta>0$. Assume that for some $i \in\{1, \ldots, k-2\}$

$$
\begin{aligned}
& \left\|\left(u(t), u_{t}(t)\right)\right\|_{\mathcal{E}_{\beta_{i}}} \\
& \quad \leq R \text { for } t \in[0, \infty) \text { and } \int_{t_{0}}^{t_{0}+h}\left\|p_{\varepsilon}(t, u)\right\|_{H^{\beta_{i}}} d t \\
& \leq C(h, R) \text { for } t_{0} \in[0, \infty) .
\end{aligned}
$$

From Lemma 5.5 we see that

$$
u \in L^{4}\left(t_{0}, t_{0}+h ; W^{\beta_{i}, 12}\right), \quad\|u\|_{L^{4}\left(t_{0}, t_{0}+h ; W^{\beta_{i}, 12}\right)} \leq C(h, R) .
$$

By the Sobolev embeding $W^{\beta_{i}, 10} \hookrightarrow L^{\frac{30}{3-10 \beta_{i}}}$ and by interpolation we see that

$$
\begin{aligned}
\|u\| & L^{5}\left(t_{0}, t_{0}+h ; L^{\frac{30}{3-10 \beta_{i}}}\right) \\
\leq & \|u\|_{L^{5}\left(t_{0}, t_{0}+h ; W^{\beta_{i}}, 10\right)} \\
\leq & \|u\|_{L^{4}\left(t_{0}, t_{0}+h ; W^{\beta_{i}, 12}\right)}^{\frac{4}{5}}\|u\|_{L_{\left(t_{0}, t_{0}+h ; H_{0}^{\beta_{i}+1}\right)}^{\frac{1}{5}} \leq C(h, R) .}
\end{aligned}
$$


Using (6.1) with $q=\frac{6}{3-10 \beta_{i}}$ we obtain

$$
\begin{aligned}
& \int_{t_{0}}^{t_{0}+h}\left\|p_{\varepsilon}(t, u)\right\|_{H^{\beta_{i+1}}} d t \\
& \left.\quad \leq C(R)\left(\|u\|_{L^{4}\left(t_{0}, t_{0}+h ; L^{12}\right)}^{4}+\|u\|^{5} L_{L_{0}, t_{0}+h ; L^{3-10 \beta_{i}}}\right)^{+h}\right) \\
& \quad \leq C(h, R),
\end{aligned}
$$

with $\beta_{i+1}=\frac{5}{2} \beta_{i}$. From this recurrent relation and the fact that $\beta_{1} \in(0, \delta)$ we can find sequence $\beta_{1}, \ldots, \beta_{k-1}$ such that $\beta_{k-1}=\frac{9}{20}$. From this exponent we can pass to $\beta_{k}=1$ in one final step. Indeed, if $\beta_{k-1}=\frac{9}{20}$, then from the Sobolev embeddings $H_{0}^{1+\frac{9}{20}} \hookrightarrow L^{60}$ and $H_{0}^{1+\frac{9}{20}} \hookrightarrow W_{0}^{1, \frac{60}{21}}$ we get the bounds

$$
\|u\|_{L^{60}} \leq C(R) \text { and }\|\nabla u\|_{L \frac{60}{21}} \leq C(R) .
$$

Hence,

$$
\left\|\nabla p_{\varepsilon}(t, u)\right\|_{L^{2}} \leq C\left(1+\int_{\Omega}|u|^{8}|\nabla u|^{2} d x\right) \leq C\left(1+\|u\|_{L^{28}}^{8}\|\nabla u\|_{L^{\frac{60}{21}}}^{2}\right) \leq C(R),
$$

and consequently it holds that

$$
\int_{t_{0}}^{t_{0}+h}\left\|\nabla p_{\varepsilon}(t, u)\right\|_{L^{2}} d t \leq C(h, R)
$$

The proof follows by the decomposition argument. Indeed, let us decompose $u(t)=$ $w(t)+v(t)$ where $w, v$ satisfy the problems

$$
\left\{\begin{array} { l } 
{ v _ { t t } + v _ { t } - \Delta v = 0 , } \\
{ v ( t , x ) = 0 \text { for } x \in \partial \Omega , } \\
{ v ( 0 , x ) = u _ { 0 } ( x ) , } \\
{ v _ { t } ( 0 , x ) = u _ { 1 } ( x ) , }
\end{array} \quad \left\{\begin{array}{l}
w_{t t}+w_{t}-\Delta w=p_{\varepsilon}(t, v+w), \\
w(t, x)=0 \text { for } x \in \partial \Omega \\
w(0, x)=0 \\
w_{t}(0, x)=0
\end{array}\right.\right.
$$

From Lemma 5.5 we get that $\left\|\left(v(t), v_{t}(t)\right)\right\|_{\mathcal{E}_{\beta_{i}}} \leq C\left\|\left(u_{0}, u_{1}\right)\right\|_{\mathcal{E}_{\beta_{i}}} e^{-\alpha t}$ and

$$
\left\|\left(w(t+h), w_{t}(t+h)\right)\right\|_{\mathcal{E}_{\beta_{i+1}}} \leq C e^{-\alpha h}\left\|\left(w(t), w_{t}(t)\right)\right\|_{\mathcal{E}_{\beta_{i+1}}}+C(h, R),
$$

for every $t \geq 0$ and $h>0$. We set $h$ such that $C e^{-\alpha h} \leq \frac{1}{2}$. Then we obtain that $\left\|\left(w(t), w_{t}(t)\right)\right\|_{\mathcal{E}_{\beta_{i+1}}} \leq 2 C(h, R)=C_{R}$ for $i \in\{0, \ldots, k-1\}$. We stress that all constants are independent of $p_{\varepsilon} \in \mathcal{H}_{[0,1]}$. 
The bounds obtained in the previous lemma allow us to deduce the asymptotic compactness of the considered non-autonomous dynamical system.

Proposition 6.9 For every $\varepsilon \in[0,1]$, the non-autonomous dynamical system $\left(\varphi_{\varepsilon}, \theta\right)$ is uniformly asymptotically compact.

Proof Let $B_{0}$ be an absorbing set from Proposition 4.5. Then for every bounded set $B \subset \mathcal{E}$ there exist $t_{0}$ such that for every $t \geq t_{0}$ and every $p_{\varepsilon} \in \mathcal{H}\left(f_{\varepsilon}\right)$ it holds that $\varphi_{\varepsilon}\left(t, p_{\varepsilon}\right) \in B_{0}$. From Lemma 6.8 there exists the set $B_{\beta_{1}} \subset \mathcal{E}_{\beta_{1}}$ which is compact in $\mathcal{E}_{0}$ such that

$$
\lim _{t \rightarrow \infty} \sup _{p_{\varepsilon} \in \mathcal{H}\left(f_{\varepsilon}\right)} \operatorname{dist}_{\mathcal{E}_{0}}\left(\varphi\left(t, p_{\varepsilon}\right) B, B_{\beta_{1}}\right)=0
$$

which shows that the non-autonomous dynamical system $\left(\varphi_{\varepsilon}, \theta\right)$ is uniformly asymptotically compact.

We are in position to formulate the main result of this section, the theorem on nonautonomous attractors.

Theorem 6.10 For every $\varepsilon \in[0,1]$ problem (3.1) has uniform $\mathcal{A}_{\varepsilon}$, cocycle $\left\{\mathcal{A}_{\varepsilon}(p)\right\}_{p \in \mathcal{H}\left(f_{\epsilon}\right)}$ and pullback attractors which are bounded in $\mathcal{E}_{1}$ uniformly with respect to $\varepsilon$. Moreover the following holds

$$
\mathcal{A}_{\varepsilon}=\bigcup_{p \in \mathcal{H}\left(f_{\epsilon}\right)} \mathcal{A}_{\varepsilon}(p) .
$$

Proof Because $\left(\varphi_{\varepsilon}, \theta\right)$ is asymptotically compact, from Theorem 6.5 we get existence of uniform and cocycle attractors and the relation between them. For $\left(u_{0}, u_{1}\right) \in \mathcal{A}_{\varepsilon}$ by Theorem 6.7 there exists the global solution $u(t)$ with $\left(u(0), u_{t}(0)\right)=\left(u_{0}, u_{1}\right)$. If $\mathcal{A}_{\varepsilon}$ is bounded in $\mathcal{E}_{\beta_{i}}$ then from Lemma 6.8 we can split this solution into the sum $u(t)=v^{n}(t)+w^{n}(t)$ for $t \in[-n, \infty)$ such that

$$
\left\|\left(v^{n}(t), v_{t}^{n}(t)\right)\right\|_{\mathcal{E}_{\beta_{i}}} \leq C e^{-\alpha(t+n)} \text { and }\left\|\left(w^{n}(t), w_{t}^{n}(t)\right)\right\|_{\mathcal{E}_{\beta_{i+1}}} \leq C .
$$

Then, for the subsequence, it holds that $w^{n}(0) \rightarrow w$ and $v^{n}(0) \rightarrow 0$ as $n \rightarrow \infty$ for some $w \in \mathcal{E}_{\beta_{i+1}}$, so $w=\left(u_{0}, u_{1}\right)$. Because $\mathcal{A}_{\uparrow}$ is bounded in $\mathcal{E}_{0}$ in finite number of steps we obtain the boudedness of the uniform attractors in $\mathcal{E}_{1}$. Moreover, due to Proposition 4.5 and Lemma 6.8 the $\mathcal{E}_{1}$ bound of these attractors does not depend on $\varepsilon$.

\section{Upper-Semicontinuous Convergence of Attractors}

The paper is concluded with the result on upper-semicontinuous convergence of attractors. 


\subsection{Definition and Properties of Upper-Semicotinuous Convergence of Sets}

We recall the definitions of Hausdorff and Kuratowski Upper-Semicontinuous convergence of sets, and the relation between these conditions.

Definition 7.1 Let $(X, d)$ be a metric space and let $\left\{A_{\varepsilon}\right\}_{\varepsilon \in[0,1]}$ be a family of sets in $X$. We say that this family converges to $A_{0}$ upper-semicontinuously in Hausdorff sense if

$$
\lim _{\varepsilon \rightarrow 0^{+}} \operatorname{dist}_{X}\left(A_{\varepsilon}, A_{0}\right)=0
$$

Definition 7.2 Let $(X, d)$ be a metric space and let $\left\{A_{\varepsilon}\right\}_{\varepsilon \in[0,1]}$ be a family of sets in $X$. We say that this family converges to $A_{0}$ upper-semicontinuously in Kuratowski sense if

$$
X-\limsup _{\varepsilon \rightarrow 0^{+}} A_{\varepsilon} \subset A_{0},
$$

where $X-\lim \sup _{\varepsilon \rightarrow 0^{+}} A_{\varepsilon}$ is the Kuratowski upper limit defined by

$$
X-\limsup _{\varepsilon \rightarrow 0^{+}} A_{\varepsilon}=\left\{x \in X: \lim _{\varepsilon_{n} \rightarrow 0^{+}} d\left(x_{\varepsilon_{n}}, x\right)=0, x_{\varepsilon_{n}} \in A_{\varepsilon_{n}}\right\} .
$$

The proof of the next result can be found for example in [17, Proposition 4.7.16].

Lemma 7.3 Assume that the sets $\left\{A_{\varepsilon}\right\}_{\varepsilon \in[0,1]}$ are nonempty and closed and the set $\cup_{\varepsilon \in[0,1]} A_{\varepsilon}$ is relatively compact in $X$. If the family $\left\{A_{\varepsilon}\right\}_{\varepsilon \in[0,1]}$ converges to $A_{0}$ upper-semicontinuously in Kuratowski sense then $\left\{A_{\varepsilon}\right\}_{\varepsilon \in[0,1]}$ converges to $A_{0}$ uppersemicontinuously in Hausdorff sense.

\subsection{Upper-Semicontinuous Convergence of Uniform Attractors}

We conclude with the result on upper-semicontinuous convergence of uniform attractors. Note that it is enough to obtain this property for the uniform attractors and the upper-semicontinuous convergence for cocycle and pullback attractors is a simple consequence.

Theorem 7.4 The family of uniform attractors $\left\{\mathcal{A}_{\varepsilon}\right\}_{\varepsilon \in[0,1]}$ for the considered nonautonomous dynamical system $\left(\varphi_{\varepsilon}, \theta_{t}\right)$ is upper-semicontinuous in Kuratowski and Hausdorff sense in $\mathcal{E}_{0}$ as $\varepsilon \rightarrow 0^{+}$.

Proof Let $\left(u_{0}^{n}, u_{1}^{n}\right) \in \mathcal{A}_{\varepsilon_{n}}$ such that $\left(u_{0}^{n}, u_{1}^{n}\right) \rightarrow\left(u_{0}, u_{1}\right)$ in $\mathcal{E}_{0}$. There exists a function $p_{\varepsilon_{n}} \in \mathcal{H}_{[0,1]}$ such that there exists global solution $u_{n}(t, x)$ to problem

$$
\left\{\begin{array}{l}
u_{t t}^{n}+u_{t}^{n}-\Delta u^{n}=p_{\varepsilon_{n}}(t, u) \\
u^{n}(t, x)=0 \text { for } x \in \partial \Omega \\
u^{n}(0, x)=u_{0}^{n}(x) \\
u_{t}^{n}(0, x)=u_{1}^{n}(x)
\end{array}\right.
$$


As in the proof of Proposition 5.13 it follows that for every $T>0$ there exists $v \in L^{\infty}\left(-T, T ; H_{0}^{1}\right)$ with $v_{t} \in L^{\infty}\left(-T, T ; L^{2}\right), v_{t t} \in L^{\infty}\left(-T, T ; H^{-1}\right)$ and $v \in$ $L^{4}\left(-T, T ; L^{12}\right)$ such that for the subsequence of $u^{n}$ there hold the convergences

$$
\begin{gathered}
u^{n} \rightarrow v \text { weakly-* in } L^{\infty}\left(-T, T ; H_{0}^{1}\right), \\
u_{t}^{n} \rightarrow v_{t} \text { weakly-* in } L^{\infty}\left(-T, T ; L^{2}\right), \\
u_{t t}^{n} \rightarrow v_{t t} \text { weakly-* in } L^{\infty}\left(-T, T ; H^{-1}\right) .
\end{gathered}
$$

Moreover $\left(u^{n}(t), u_{t}^{n}(t)\right) \rightarrow\left(v(t), v_{t}(t)\right)$ weakly in $\mathcal{E}_{0}$ for every $t \in[-T, T]$ which implies that $\left(v(0), v_{t}(0)\right)=\left(u_{0}, u_{1}\right)$ and $u^{n}(t) \rightarrow v(t)$ strongly in $L^{2}$. We will show that $v$ is a weak solution for the autonomous problem, i.e., the problem with $\varepsilon=0$. It is enough to show that for every $w \in L^{2}\left(-T, T ; H_{0}^{1}\right)$ it holds that

$$
\lim _{n \rightarrow \infty} \int_{-T}^{T}\left(p_{\varepsilon_{n}}\left(t, u^{n}(t)\right)-f_{0}\left(v^{n}(t)\right), w(t)\right) d t=0 .
$$

Let observe that $\left\|u_{n}(t)\right\|_{C^{0}} \leq R$ and $\|v(t)\|_{C^{0}} \leq R$ due to the fact that all attractors are bounded uniformly in $\mathcal{E}_{1}$ and the Sobolev embedding $H^{2} \hookrightarrow C^{0}$. Hence

$$
\begin{aligned}
& \left|\int_{-T}^{T}\left(p_{\varepsilon_{n}}\left(t, u^{n}(t)\right)-f_{0}(v(t)), w(t)\right) d t\right| \\
& \quad \leq \int_{-T}^{T}\left|\left(p_{\varepsilon_{n}}\left(t, u^{n}(t)\right)-f_{0}\left(u^{n}(t)\right), w(t)\right)\right| d t \\
& \quad+\int_{-T}^{T}\left|\left(f_{0}\left(u^{n}(t)\right)-f_{0}(v(t)), w(t)\right)\right| d t \\
& \quad \leq \sup _{t \in \mathbb{R}} \sup _{|s| \leq R}\left|\left(p_{\varepsilon_{n}}(t, s)-f_{0}(s)\right)\right|\|w\|_{L^{1}\left(-T, T ; L^{2}\right)} \\
& \quad+\sup _{|s| \leq R}\left|f_{0}^{\prime}(s)\right|\left(\int_{-T}^{T}\left\|u^{n}(t)-v(t)\right\|^{2} d t\right)^{\frac{1}{2}}\|w\|_{L^{2}\left(-T, T ; L^{2}\right)}^{\frac{1}{2}}
\end{aligned}
$$

Due to the fact that by Proposition 3.6 the hypothesis (H2) holds for $p_{\varepsilon}$, the first term tends to zero. The second term tends to zero by the Aubin-Lions lemma. Hence, $v(t)$ is the weak solution on the interval $[-T, T]$ with $v(0)=\left(u_{0}, u_{1}\right)$. By the diagonal argument we can extend $v$ to a global weak solution. Moreover $\|v(t)\|_{\mathcal{E}_{1}} \leq C$ due to the uniform boudedness of attractors $\mathcal{A}_{\varepsilon}$ in $\mathcal{E}_{1}$. Hence $\{v(t)\}_{t \in \mathbb{R}}$ is a global bounded orbit for the autonomous dynamical system $\varphi_{0}$ which implies that $\left(u_{0}, v_{0}\right) \in \mathcal{A}_{0}$ and shows the upper-semicontinuity in the Kuratowski sense. Because all uniform attractors $\mathcal{A}_{\varepsilon}$ are uniformly bounded in $\mathcal{E}_{1}$, their union $\cup_{\varepsilon \in[0,1]} \mathcal{A}_{\varepsilon}$ is relatively compact in $\mathcal{E}_{0}$. So, by Lemma 7.3 we have also upper-semicontinuity in Hausdorff sense.

Open Access This article is licensed under a Creative Commons Attribution 4.0 International License, which permits use, sharing, adaptation, distribution and reproduction in any medium or format, as long as you give 
appropriate credit to the original author(s) and the source, provide a link to the Creative Commons licence, and indicate if changes were made. The images or other third party material in this article are included in the article's Creative Commons licence, unless indicated otherwise in a credit line to the material. If material is not included in the article's Creative Commons licence and your intended use is not permitted by statutory regulation or exceeds the permitted use, you will need to obtain permission directly from the copyright holder. To view a copy of this licence, visit http://creativecommons.org/licenses/by/4.0/.

\section{References}

1. Antil, H., Pfefferer, J., Rogovs, S.: Fractional operators with inhomogeneous boundary conditions: analysis, control, and discretization. Commun. Math. Sci. 16, 1395-1426 (2018)

2. Arrieta, J.M., Carvalho, A.N., Hale, J.K.: A damped hyperbolic equation with critical exponent. Comm. Partial Differ. Equ. 17, 841-866 (1992)

3. Babin, A.V., Vishik, M.I.: Regular attractors of semi-groups and evolution equations. J. Math. Pures et Appl. 62, 441-491 (1983)

4. Babin, A.V., Vishik, M.I.: Attractors of Evolution Equations, Studies in Mathematics and Its Applications, 25. North-Holland Publishing Co., Amsterdam (1992)

5. Balibrea, F., Caraballo, T., Kloeden, P.E., Valero, J.: Recent developments in dynamical systems: three perspectives. Int. J. Bifurcat. Chaos 20, 2591-2636 (2010)

6. Blair, M., Smith, H., Sogge, C.: Strichartz estimates for the wave equation on manifolds with boundary. Ann. I. H. Poincaré-AN 26, 1817-1829 (2009)

7. Bortolan, M.C., Carvalho, A.N., Langa, J.A.: Structure of attractors for skew product semiflows. J. Differ. Equ. 257(2), 490-522 (2014)

8. Bortolan, M.C., Carvalho, A.N., Langa, J.A.: Attractors Under Autonomous and Non-autonomous Perturbations. American Mathematical Society: Mathematical Surveys and Monographs, Providence (2020)

9. Burq, N., Lebeau, G., Planchon, F.: Global existence for energy critical waves in 3D domains. J. AMS 21, 831-845 (2008)

10. Carvalho, A.N., Cholewa, J.W., Dłotko, T.: Damped wave equations with fast growing dissipative nonlinearities. Discrete Cont. Dyn. Syst. A 24, 1147-1165 (2009)

11. Carvalho, A.N., Langa, J.A., Robinson, J.C.: Attractors for Infinite-Dimensional Non-autonomous Dynamical Systems, Applied Mathematical Series, 182. Springer, New York (2013)

12. Chang, Q., Li, D., Sun, C., Zelik, S.: Deterministic and random attractors for a wave equation with sign changing damping. arXiv:1910.02430

13. Cheban, D.N.: Global Attractors of Non-autonomous Dissipative Dynamical Systems. World Scientific, Singapore (2004)

14. Chepyzhov, V.V.: Uniform attractors of dynamical processes and non-autonomous equations of mathematical physics. Russ. Math. Surv. 68, 159-196 (2013)

15. Chepyzhov, V.V., Vishik, M.I.: Attractors for Equations of Mathematical Physics. American Mathematical Society, Providence (2002)

16. Chueshov, I., Lasiecka, I.: Long-Time Behavior of Second Order Evolution Equations with Nonlinear Damping, Memoirs of the American Mathematical Society, 912. American Mathematical Society, Providence (2008)

17. Denkowski, Z., Migórski, S., Papageorgiou, N.S.: An Introduction to Nonlinear Analysis: Theory. Kluwer Academic Publishers, Dordrecht (2003)

18. Dłotko, T., Cholewa, J.W.: Global Attractors in Abstract Parabolic Problems. Cambridge University Press, Cambridge (2000)

19. Freitas, M., Kalita, P., Langa, J.: Continuity of non-autonomous attractors for hyperbolic perturbation of parabolic equations. J. Diff. Equ. 264, 1886 (2017)

20. Ghidaglia, J.M., Temam, R.: Attractors for damped nonlinear hyperbolic equations. J. Math. Pures et Appl. 66, 273-319 (1987)

21. Hale, J.K.: Asymptotic behavior and dynamics in infinite dimensions, 1-42, Nonlinear Differential Equations, Research Notes in Math., 132, Pitman (1985)

22. Hale, J.K.: Asymptotic Behavior of Dissipative Systems. American Mathematical Society, Mathematical Surveys and Monographs, Providence (1988) 
23. Haraux, A.: Two remarks on dissipative hyperbolic problems, Nonlinear partial differential equations and their applications, College de France Seminar, Research Notes in Math., 122, 161-179 (1984)

24. Haraux, A.: Recent results on semi-linear hyperbolic problems in bounded domains, Partial Differential Equations, Lecture Notes in Math., 1324, 118-126. Springer, Berlin, Heidelberg (1988)

25. Haraux, A.: Semi-Linear Hyperbolic Problems in Bounded Domains. CRC Press, Boca Raton (1987)

26. Henry, D.: Geometric Theory of Semilinear Parabolic Equations, Springer, Lecture Notes in Math., 840, Berlin (1981)

27. Kalantarov, V., Savostianov, A., Zelik, S.: Attractors for damped quintic wave equations in bounded domains. Ann. Henri Poincaré 17, 2555-2584 (2016)

28. Kloeden, P.E., Rasmussen, M.: Systems, Nonautonomous Dynamical. American Mathematical Society, Providence (2010)

29. Liu, C., Meng, F., Sun, C.: Well-posedness and attractors for a super-cubic weakly damped wave equation with $H^{-1}$ source term. J. Differ. Equ. 263, 8718-8748 (2017)

30. Mei, X., Sun, C.: Uniform attractors for a weakly damped wave equation with sup-cubic nonlinearity. Appl. Math. Lett. 95, 179-185 (2019)

31. Mei, X., Xiong, Y., Sun, C.: Discrete and Continuous Dynamical Systems, Pullback attractor for a weakly damped wave equation with sup-cubic nonlinearity. 41, 569-600 (2021)

32. Meng, F., Liu, C.: Exponential attractors for weakly damped wave equation with sub-quintic nonlinearity. Comput. Math. Appl. 78, 1026-1036 (2019)

33. Pata, V., Zelik, S.: A remark on the damped wave equation. Commun. Pure Appl. Anal. 611-616, 5 (2006)

34. Robinson, J.C.: Infinite-Dimensional Dynamical Systems. Cambridge University Press, Cambridge (2001)

35. Savostianov, A.: Strichartz estimates and smooth attractors for a sub-quintic wave equation with fractional damping in bounded domains. Adv. Differ. Equ. 20, 495-530 (2015)

36. Savostianov, A., Zelik, S.: Smooth attractors for the quintic wave equations with fractional damping. Asymptot. Anal. 87, 191-221 (2014)

37. Savostianov, A., Zelik, S.: Uniform attractors for measure-driven quintic wave equations. Russ. Math. Surv. 75, 253-320 (2020)

38. Smith, H.F., Sogge, C.D.: Global Strichartz estimates for nonthapping perturbations of the Laplacian. Commun. Partial Differ. Equ. 25, 2171-2183 (2000)

39. Simon, J.: Compact sets in the space $L^{p}(0, T ; B)$. Ann. di Matematica 146, 65-96 (1986)

40. Tao, T.: Nonlinear dispersive equations: local and global analysis. In: Proceedings of the CBMS Regional Conference Series in Mathematics (2006)

41. Temam, R.: Infinite-Dimensional Dynamical Systems in Mechanics and Physics. Springer, New York (1988)

Publisher's Note Springer Nature remains neutral with regard to jurisdictional claims in published maps and institutional affiliations. 